\title{
Processamento, propriedades e aplicações das cerâmicas de nitreto de alumínio
}

\section{(Processing, properties and applications of aluminum nitride ceramics)}

\author{
A. L. Molisani \\ Faculdade de Engenharia "Engenheiro Celso Daniel”, Centro Universitário Fundação Santo André, \\ Av. Príncipe de Gales 821, Santo André, SP 09060-650 \\ andre.molisani@fsa.br
}

\begin{abstract}
Resumo
O nitreto de alumínio (AlN) é considerado um adequado material para substratos e materiais de encapsulamento de dispositivos microeletrônicos devido à elevada condutividade térmica, às excelentes propriedades elétricas e ao coeficiente de expansão térmica próximo daquele observado para o silício. Estas propriedades tornam o $\mathrm{AlN}$ um excelente candidato para substituir a alumina $\left(\mathrm{Al}_{2} \mathrm{O}_{3}\right)$ e berília $(\mathrm{BeO})$ usadas na fabricação de dispositivos semicondutores. O presente artigo apresenta o estado da arte da cerâmica de AlN, com objetivo de mostrar os avanços em processamento desenvolvidos nas últimas décadas, visando a aplicação em dispositivos eletrônicos. Outro objetivo foi verificar a possibilidade de uso do AlN em aplicações estruturais. A cerâmica de AlN apresentou significativos avanços tecnológicos ao longo das últimas décadas, como diminuição da temperatura de síntese e de sinterização, produção de pó resistente à água e produção de substrato cerâmico pela tecnologia DBC (direct bonding copper). Porém, a maioria dos referidos avanços tecnológicos não foi introduzida nas rotas industriais de produção em larga escala. Deste modo, os custos de produção não foram reduzidos suficientemente para tornar o $\mathrm{AlN}$ atrativo às indústrias de microeletrônica. $\mathrm{Embora} \mathrm{o} \mathrm{AlN}^{\mathrm{N}} \mathrm{Al}_{2} \mathrm{O}_{3}$ tenham propriedades mecânicas relativamente próximas, o AlN é pouco atrativo como material estrutural devido ao seu elevado custo de produção em comparação com o $\mathrm{Al}_{2} \mathrm{O}_{3}$. Portanto, desenvolvimentos prévios e atuais em processamento devem ser implantados industrialmente para promover a redução de custos, aumentando a competitividade do AlN nos mercados de cerâmicas eletrônicas e estruturais. Estes mercados também podem ser expandidos usando o AlN em novas aplicações, como proteção balística e dispositivos nanoestruturados (componentes optoeletrônicos, lasers de alta energia, sensores de onda pulsante, ressonadores mecânicos, diodos emissores de luz e emissores de elétrons por campo).
\end{abstract}

Palavras-chave: AlN, síntese, sinterização, condutividade térmica, propriedades mecânicas.

\begin{abstract}
Aluminum nitride (AlN) is expected to be suitable as substrates and packages for microelectronic devices because of its high thermal conductivity, excellent electrical properties, and thermal expansion coefficient close to that of silicon. These properties make AlN an excellent candidate to replace alumina $\left(\mathrm{Al}_{2} \mathrm{O}_{3}\right)$ and beryllia $(\mathrm{BeO})$ used for the manufacture of semiconductor devices. This paper shows the state-of-the-art in AlN ceramics, aiming to show the advances in processing developed in the last decades for the applications in electronic devices. Another objective was to verify the possibility of using AlN in structural applications. AlN ceramics exhibited significant technological advances over the past decades, as diminution in synthesis and sintering temperatures, synthesis of water resistant powder, and production of ceramic substrate by the direct bonding copper technology. However, most of these technological advances were not introduced in the industrial routes for large-scale production. As a result, the production costs were not reduced sufficiently to make AlN attractive for the microelectronic industries. Although $\mathrm{AlN}_{\mathrm{N}}$ and $\mathrm{Al}_{2} \mathrm{O}_{3}$ have relatively close mechanical properties, $\mathrm{AlN}$ is unattractive as a structural material because of its high production cost in comparison to $\mathrm{Al}_{2} \mathrm{O}_{3}$. Therefore, previous and current developments in processing must be implemented industrially to promote cost reduction, increasing the competitiveness of AlN in the electronic and structural ceramics markets. These markets can also be expanded using AlN in new applications such as armor products and nanostructured devices (optoelectronic components, high-energy lasers, pulse wave sensors, mechanical resonators, light-emitting diodes, and field electron emitters).
\end{abstract}

Keywords: AlN, synthesis, sintering, thermal conductivity, mechanical properties.

\section{INTRODUÇÃO}

A tecnologia planar em silício possibilitou a fabricação de dispositivos eletrônicos miniaturizados (circuitos integrados ou chips). Este processo avançou até atingir dimensões que resultaram em problemas tanto de flexibilização funcional como de custos efetivos, que inviabilizaram a produção de chips com alta complexidade. Os referidos problemas foram solucionados com o advento da tecnologia de circuitos passivos, que é responsável pela fabricação de substratos e componentes para encapsulamento (packaging), os quais são acoplados ao chip de silício para fornecer interconexão, 
sustentação e proteção [1]. A redução de tamanho do chip promove, naturalmente, $\mathrm{o}$ adensamento de seus componentes eletrônicos ativos (capacitores, resistores, transistores e outros), o que pode gerar elevada dissipação térmica, quando o dispositivo é submetido a condições de trabalho em alta potência $[2,3]$. Nestas condições, o gerenciamento térmico é um fator chave para a confiabilidade, o desempenho e a miniaturização dos dispositivos microeletrônicos [4]. Uma solução para aumentar a eficiência em dissipação térmica é o uso de materiais com elevada condutividade térmica, que atuam como absorvedores de calor (heat sinks), evitando falhas por superaquecimento [5]. Contudo, os materiais que compõem os dispositivos eletrônicos precisam ter valores próximos de coeficiente de expansão térmica para evitar falhas de curto-circuito devido ao rompimento das trilhas condutoras por diferenças de dilatação térmica [4]. Os dispositivos microeletrônicos de alta potência possuem limitado campo de aplicação porque os substratos e componentes para encapsulamento não fornecem condições adequadas de isolamento, interconexão e gerenciamento térmico em operações de alta temperatura $\left(\leq 300{ }^{\circ} \mathrm{C}\right)$ sob ambientes severos [6]. Para aumentar a eficiência dos referidos módulos de potência, almejam-se circuitos passivos com as seguintes propriedades: boa resistência mecânica para suportar o chip e os processos de metalização; alta resistividade para isolar eletricamente o chip; estabilidade química para evitar reações com os demais componentes eletrônicos; baixa constante dielétrica para diminuir perdas por micro-ondas; e bom acabamento superficial para facilitar a deposição de filmes espessos [6].

A tecnologia de circuitos passivos desenvolveu duas rotas de processamento para módulos de potência, que operam em elevadas temperaturas e ambientes severos. A primeira rota de processamento é denominada cerâmica cossinterizada em alta temperatura (high temperature cofired ceramic, HTCC), que consiste basicamente em aplicar multicamadas de filmes espessos, com composição química variável, sobre a superfície de um substrato cerâmico não sinterizado produzido por colagem de fita (tape casting) [7-9]. Em seguida, vários substratos cerâmicos com diferentes sistemas multicamadas são sobrepostos e, então, submetidos aos processos de alinhamento, usinagem, furação, metalização e laminação, o que permite a construção de vários tipos de estruturas 3D [7, 9]. A estrutura 3D laminada é sinterizada entre 1500 e $1600{ }^{\circ} \mathrm{C}$, o que resulta em um único módulo eletrônico denominado circuito híbrido [8]. Dispositivos eletrônicos fabricados pela tecnologia HTCC são usados em indústrias automobilísticas, sistemas de comunicação por rede sem fio (wireless) e equipamentos de radiofrequência [7]. A segunda rota de processamento para módulos de potência é denominada colagem direta de cobre (direct bonding copper, DBC) [9]. Este processo inicia-se com a oxidação da superfície de uma lâmina de cobre (formação de $\mathrm{CuO}_{2}$ ), que é subsequentemente colada na superfície de um substrato cerâmico sinterizado por meio de tratamento térmico em altas temperaturas [5]. Em cerâmicas óxidas (por ex., alumina $-\mathrm{Al}_{2} \mathrm{O}_{3}$ ), o tratamento térmico promove, na região de interface, a formação de um composto eutético $\left(\mathrm{CuAl}_{2} \mathrm{O}_{4}\right)$ devido à reação do sistema $\mathrm{CuO}_{2}-\mathrm{Al}_{2} \mathrm{O}_{3}$, sendo a respectiva fase eutética responsável pela adesão metalcerâmica $[5,10]$. Em relação às cerâmicas não óxidas (por ex., nitreto de alumínio - AlN), o processo DBC exibe uma

Tabela I - Propriedades térmicas, elétricas e físicas das cerâmicas de $\mathrm{AlN}, \mathrm{Al}_{2} \mathrm{O}_{3}$ e $\mathrm{BeO}$ usadas na indústria de microeletrônica [18].

[Table I-Thermal, electrical, and physical properties of $\mathrm{AlN}, \mathrm{Al}_{2} \mathrm{O}_{3}$ and $\mathrm{BeO}$ ceramics used in the microelectronic industry [18].]

\begin{tabular}{cccc}
\hline Propriedade & $\mathrm{AlN}$ & $\mathrm{Al}_{2} \mathrm{O}_{3}$ & $\mathrm{BeO}$ \\
\hline Térmica & & & \\
Condutividade térmica a $25^{\circ} \mathrm{C}(\mathrm{W} / \mathrm{mK})$ & $170-220$ & $20-30$ & $250-300$ \\
Coeficiente de expansão térmica entre 25 e $400{ }^{\circ} \mathrm{C}\left(\mathrm{x} 10^{-6}{ }^{\circ} \mathrm{C}^{-1}\right)$ & $4,3-4,6$ & $6,7-7,3$ & $7,5-8,0$ \\
Resistência ao choque térmico, $\Delta \mathrm{Tc}\left({ }^{\circ} \mathrm{C}\right)$ & 550 & 150 & - \\
\hline Elétrica & & & \\
Constante dielétrica a $25^{\circ} \mathrm{C}$ e $1 \mathrm{MHz}$ & $8,8-8,9$ & $8,8-8,9$ & $6,5-6,7$ \\
Perda dielétrica a $25^{\circ} \mathrm{C}$ e $1 \mathrm{MHz}\left(\mathrm{x} 10^{-4}\right)$ & $1-5$ & $2-3$ & $1-5$ \\
Resistividade elétrica a $25^{\circ} \mathrm{C}(\Omega \cdot \mathrm{cm})$ & $>10^{14}$ & $>10^{14}$ & $>10^{14}$ \\
Rigidez dielétrica a $25^{\circ} \mathrm{C}(\mathrm{kV} / \mathrm{cm})$ & $140-170$ & 100 & 100 \\
\hline Física & & & \\
Densidade $\left(\mathrm{kg} / \mathrm{m}^{3}\right)$ & 3260 & 3970 & 3010 \\
Resistência à flexão $(\mathrm{MPa})$ & $400-500$ & 300 & 250 \\
Dureza Vickers $(\mathrm{GPa})$ & 12,0 & 25,0 & 12,0 \\
Módulo de Young $(\mathrm{GPa})$ & 343 & 378 & 378 \\
\hline
\end{tabular}


etapa adicional, que envolve a pré-oxidação da superfície do substrato de $\mathrm{AlN}$, com formação de uma camada de $\mathrm{Al}_{2} \mathrm{O}_{3}$ $[5,11]$. Neste caso, a adesão metal-cerâmica ocorre devido à formação do $\mathrm{CuAl}_{2} \mathrm{O}_{4}$, similar ao observado no processo $\mathrm{DBC}$ para $\mathrm{o}_{2} \mathrm{Ol}_{3}$ [5]. Nos últimos anos, observaram-se estudos sobre a utilização da tecnologia DBC na fabricação de diodos emissores de luz (LED, light emitting diode) [5, 12]. No caso dos módulos de LED de alta potência, aspiramse materiais de encapsulamento com elevada condutividade térmica, pois o LED não irradia calor paro o exterior, como observado na lâmpada de bulbo convencional [5].

Os processos HTCC e DBC foram desenvolvidos industrialmente usando alumina como substrato cerâmico $[5,10]$. Habitualmente, este substrato é processado com $90 \%$ a $96 \%$ em massa de $\mathrm{Al}_{2} \mathrm{O}_{3}$, sendo adicionados $4 \%$ a $10 \%$ em massa de um composto do sistema $\mathrm{SiO}_{2}-\mathrm{MgO}-\mathrm{CaO}$ para promover a densificação entre 1400 e $1500^{\circ} \mathrm{C}$ (viabilização do processo HTCC) e melhorar a aderência metal-cerâmica (otimização do processo DBC) [12]. Apesar de sua ampla comercialização, $\mathrm{o} \mathrm{Al}_{2} \mathrm{O}_{3}$ tem baixa condutividade térmica (20-24 W/mK à temperatura ambiente), bem como elevado coeficiente de expansão térmica $(6,9-7,2 \times 10$ $\left.{ }^{6}{ }^{\circ} \mathrm{C}^{-1}\right)$ em relação ao chip de silício $\left(3,2 \times 10^{-6}{ }^{\circ} \mathrm{C}^{-1}\right)$ [13]. Sendo assim, $\mathrm{o} \mathrm{Al}_{2} \mathrm{O}_{3}$ não é recomendado para módulos de potência ou qualquer aplicação que necessite de alta dissipação térmica. Na indústria de microeletrônica, cogitou-se a substituição do $\mathrm{Al}_{2} \mathrm{O}_{3}$ por berília $(\mathrm{BeO})$ devido à sua elevada condutividade térmica $(240-260 \mathrm{~W} / \mathrm{mK}$ à temperatura ambiente) [1], mas logo caiu em desuso por causa da toxidade e elevado coeficiente de expansão térmica $\left(8 \times 10^{-6}{ }^{\circ} \mathrm{C}^{-1}\right)$ [14]. O AlN é outra cerâmica que despertou o interesse da indústria de microeletrônica, principalmente a partir da década de 1990, quando se verificou que o monocristal de AlN isento de oxigênio pode alcançar condutividade térmica de $320 \mathrm{~W} / \mathrm{mK}$ à temperatura ambiente - cerca de $80 \%$ da condutividade térmica do cobre metálico [15]. Esta cerâmica foi considerada excelente candidata para substituir $\mathrm{o} \mathrm{Al}_{2} \mathrm{O}_{3}$ e $\mathrm{BeO}$ na fabricação de dispositivos eletrônicos com elevada dissipação térmica [2, $13,16]$. Além da elevada condutividade térmica $(80-200 \mathrm{~W} /$ $\mathrm{mK}$ ) [16], o AlN exibe outras propriedades relevantes para a fabricação de dispositivos eletrônicos, quais sejam [13, 17]: elevada resistência à ruptura dielétrica, baixa perda de energia em alta frequência, elevada resistividade elétrica e coeficiente de expansão térmica $\left(4,4 \times 10^{-6}{ }^{\circ} \mathrm{C}^{-1}\right)$ próximo daquele observado para o silício $\left(3,2 \times 10^{-6}{ }^{\circ} \mathrm{C}^{-1}\right)$. A Tabela I apresenta um comparativo entre as principais propriedades térmicas, elétricas e físicas das cerâmicas de $\mathrm{AlN}, \mathrm{Al}_{2} \mathrm{O}_{3} \mathrm{e}$ $\mathrm{BeO}$ usadas na fabricação de substratos e componentes para encapsulamento de módulos de alta potência.

No início da década de 1990, época em que o AlN foi indicado para as novas gerações de dispositivos eletrônicos com elevada dissipação térmica [17], a empresa Dow Chemical reportou que a rápida aceitação do AlN na indústria eletrônica seria restringida pelos seguintes fatores [19]: i) elevado custo de produção do pó cerâmico - preço médio de $110 \mathrm{US} \$ / \mathrm{kg}$ para o $\mathrm{AlN}$ e de $5 \mathrm{US} \$ / \mathrm{kg}$ para o $\mathrm{Al}_{2} \mathrm{O}_{3}$; ii) indisponibilidade de materiais com propriedades previsíveis e consistentes; iii) inexperiência em processamento cerâmico; e iv) incertezas em relação às fontes confiáveis de abastecimento. Sendo assim, o presente artigo apresenta o estado da arte da cerâmica de AlN, com o objetivo de mostrar os avanços em processamento desenvolvidos nas últimas décadas, visando a aplicação em dispositivos eletrônicos. Adicionalmente, objetivou-se verificar a possibilidade de uso do AlN em aplicações estruturais, como observado para as cerâmicas de $\mathrm{Al}_{2} \mathrm{O}_{3}$, nitreto de silício $\left(\mathrm{Si}_{3} \mathrm{~N}_{4}\right)$ e carbeto de silício $(\mathrm{SiC})$.

\section{SÍNTESE E HIDRÓLISE DO NITRETO DE ALUMÍNIO}

O AlN foi descoberto em 1862, mas a primeira síntese ocorreu apenas em 1876 [20]. Esta cerâmica não exibe ponto de fusão, pois se dissocia em alumínio $(\mathrm{Al})$ e nitrogênio $(\mathrm{N})$ acima de $2230{ }^{\circ} \mathrm{C}$ [20]. Embora seja considerado covalente [13], o AlN é constituído por ligações tanto covalente (60\%) como iônica (40\%) [21], tendo estrutura cristalina hexagonal compacta $2 \mathrm{H}$ similar à da wurtzita $[13,20]$. A cerâmica de AlN é sintetizada a partir de matérias-primas industrializadas, como pós de alumínio (Al) ou de $\mathrm{Al}_{2} \mathrm{O}_{3}$. Embora haja vários métodos de síntese do pó de AlN, tais como deposição química a vapor [22], plasma [23] e pirólise a partir de compostos à base de alumínio $\left[\mathrm{Al}(\mathrm{NO})_{3}, \mathrm{Al}(\mathrm{OH})_{3}\right.$, $\left.\mathrm{Al}(\mathrm{OH}) .\left(\mathrm{O}_{2} \mathrm{CCH} .\left(\mathrm{C}_{2} \mathrm{H}_{5}\right) \cdot\left(\mathrm{C}_{4} \mathrm{H}_{9}\right)\right)_{2}\right]$ [24], os métodos usuais na indústria são redução carbotérmica e nitretação (RCN) do pó de $\mathrm{Al}_{2} \mathrm{O}_{3}$ e nitretação direta (ND) do pó de $\mathrm{Al}$ [13]. O processo RCN é mais atrativo do que o processo ND, pois resulta em pó de AlN com alta pureza, elevada resistência à degradação por umidade e elevada sinterabilidade $[25,26]$.

Tabela II - Características técnicas dos pós de AlN sintetizados por redução carbotérmica e nitretação $(\mathrm{RCN})$ do pó de $\mathrm{Al}_{2} \mathrm{O}_{3}$ (Tokuyama Soda, Japão) e por nitretação direta (ND) do pó de alumínio (H.C. Starck, Alemanha) [30].

[Table II - Technical characteristics of synthesized AlN powders by carbothermal reduction and nitridation of $\mathrm{Al}_{2} \mathrm{O}_{3}$ powder (Tokuyama Soda, Japan), and by direct nitridation of aluminum powder (H.C. Starck, German) [30].]

\begin{tabular}{ccccc}
\hline \multirow{2}{*}{ Característica } & \multicolumn{2}{c}{ RCN } & \multicolumn{2}{c}{ ND } \\
& Grau F & Grau H & Grau B & Grau C \\
\hline $\begin{array}{c}\text { Área de superfície } \\
\text { específica }\left(\mathrm{m}^{2} / \mathrm{g}\right)\end{array}$ & 3,31 & 2,63 & 2,80 & 4,00 \\
Tamanho médio & 1,31 & 1,55 & 3,25 & 2,41 \\
de partícula $(\mu \mathrm{m})$ & & & & \\
O (\% massa) & 0,89 & 0,83 & 1,14 & 1,60 \\
$\mathrm{C}(\%$ massa) & 0,04 & 0,02 & 0,05 & 0,04 \\
$\mathrm{Si}$ (ppm massa) & $<9$ & 38 & 0 & 0 \\
$\mathrm{Fe}$ (ppm massa) & $<10$ & 10 & $<20$ & 20 \\
$\mathrm{Ca}$ (ppm massa) & 6 & 220 & 0 & 0 \\
\hline
\end{tabular}


Neste processo, pós de $\mathrm{Al}_{2} \mathrm{O}_{3}$ e carbono (C) são misturados em meio aquoso ou em solvente orgânico para obter uma mistura homogênea [27]. Após a secagem, a mistura homogeneizada é tratada termicamente em forno com fluxo de gás nitrogênio ou amônia $\left(\mathrm{Al}_{2} \mathrm{O}_{3}+3 \mathrm{C}+\mathrm{N}_{2} \rightarrow \mathrm{AlN}+3 \mathrm{CO}\right)$ entre 1400 e $1700{ }^{\circ} \mathrm{C}[27,28]$. Uma subsequente calcinação ao ar ou em atmosfera oxidante é realizada entre 400 e 800 ${ }^{\circ} \mathrm{C}$ para remoção do $\mathrm{C}$ não reagido [27]. A calcinação não deve ser realizada acima de $800{ }^{\circ} \mathrm{C}$ devido ao problema de oxidação do pó de AlN [27]. Apesar da temperatura de fusão do $\mathrm{Al}$ ser ao redor $660^{\circ} \mathrm{C}$, o processo ND é realizado em temperaturas tão altas quanto $1150{ }^{\circ} \mathrm{C}[27,29]$. O pó de $\mathrm{Al}$ é fundido em altas temperaturas e, então, injetase gás nitrogênio ou amônia para promover a nitretação $\left(2 \mathrm{Al}+\mathrm{N}_{2} \rightarrow 2 \mathrm{AlN}\right)$. O AlN sintetizado por ND deve ser submetido a um processo de moagem, o que pode resultar no aumento do teor de oxigênio presente na superfície do pó de partida [13]. A Tabela II mostra algumas características técnicas dos pós de AlN produzidos pelos processos RCN e ND.

Os custos de produção do pó de AlN são dispendiosos devido às elevadas temperaturas de síntese [26, 28]. Entretanto, a temperatura de síntese do AlN pelo processo RCN pode ser diminuída para valores tão baixos quanto $1300{ }^{\circ} \mathrm{C}$ por meio da adição de agentes catalizadores $\left(\mathrm{CaF}_{2}\right.$, $\mathrm{CaF}_{2}-\mathrm{SrCO}_{3}, \mathrm{CaF}_{2}-\mathrm{Li}_{2} \mathrm{CO}_{3}$ e $\mathrm{CaF}_{2}-\mathrm{Y}_{2} \mathrm{O}_{3}-\mathrm{Li}_{2} \mathrm{CO}_{3}$ ) na mistura $\mathrm{Al}_{2} \mathrm{O}_{3}-\mathrm{C}[31,32]$. No processo $\mathrm{ND}$, adições de sais à base de lítio $\left(\mathrm{LiNO}_{3}, \mathrm{LiOH} . \mathrm{H}_{2} \mathrm{O}\right.$ e $\left.\mathrm{Li}_{2} \mathrm{CO}_{3}\right)$ causam significativa diminuição na temperatura de síntese do pó de AlN (550$600{ }^{\circ} \mathrm{C}$ ) [29]. Dependendo da temperatura de síntese, os dois processos (RCN ou ND) podem resultar em pós de AlN com significativo residual de segundas fases oriundas das adições de agentes catalizadores. Deste modo, pode-se obter pós de AlN pré-aditivados, o que elimina a necessidade do uso de aditivos de sinterização [32].

O pó de AlN é hidrolisado ao ser exposto à umidade do meio ambiente, sendo formada uma camada de hidróxido de alumínio na sua superfície [30, 33, 34]. Quando o pó de AlN é disperso em solução aquosa, forma-se inicialmente uma camada amorfa de hidróxido de $\mathrm{Al}$ (bohemita, $\mathrm{AlOOH}$ ), a qual se transforma em bayerita cristalina $\left[\mathrm{Al}\left(\mathrm{OH}_{3}\right)\right]$ após longos períodos de exposição [33]. A camada reagida é porosa e, portanto, o processo de degradação continua até a completa hidrólise do AlN [33]. Este processo também leva à formação de amônia $\left(\mathrm{NH}_{3}\right)$, que é um produto tóxico [33]. As possíveis reações químicas que podem ocorrer durante a hidrólise do pó de AlN são [33]:

$$
\begin{aligned}
& \mathrm{AlN}+2 \mathrm{H}_{2} \mathrm{O} \leftrightarrow \mathrm{AlOOH}_{\text {amorfo }}+\mathrm{NH}_{3} \\
& \mathrm{NH}_{3}+\mathrm{H}_{2} \mathrm{O} \leftrightarrow \mathrm{NH}_{4}^{+}+\mathrm{OH}^{-} \\
& \mathrm{AlOOH}_{\text {amorfo }}+\mathrm{H}_{2} \mathrm{O} \leftrightarrow \mathrm{Al}(\mathrm{OH})_{3}
\end{aligned}
$$

Apesar da vulnerabilidade do pó de AlN em ambientes úmidos ou soluções aquosas, é possível produzir pós resistentes à água por meio de tratamento superficial com ácido esteárico ou fosfórico [35, 36], o que viabiliza a produção de substratos cerâmicos por colagem de fita em meio aquoso [35-37]. Embora haja resultados promissores em relação à diminuição da temperatura de síntese e produção de pós resistentes à água, os principais fornecedores de pós de AlN continuam utilizando as rotas tradicionais de processamento. Atualmente, a empresa Tokuyama fornece os seguintes produtos fabricados pelo processo RCN: i) pó de AlN; ii) pó granulado de AlN (contendo aditivos de sinterização, ligantes e plastificantes); e iii) pó de AlN resistente à água para uso como material de preenchimento (filler) em compósitos.

\section{SINTERIZAÇÃO DO NITRETO DE ALUMÍNIO}

O AlN é difícil de sinterizar devido à sua baixa difusividade oriunda da forte ligação covalente Al-N, sendo necessárias elevadas temperaturas de sinterização (>1900 ${ }^{\circ} \mathrm{C}$ ) para promover a densificação da cerâmica sem aditivo $[38,39]$. Outros estudos ressaltaram que o AlN é difícil de fabricar [40], pois necessita de sinterização sob pressão (hot pressing sintering) ou pós submicrométricos para densificar [41]. As referidas condições de processamento aumentam os custos de fabricação, além de impossibilitar a adequação do AlN ao processo HTCC pré-estabelecido para $\mathrm{O}_{2} \mathrm{Al}_{2} \mathrm{O}_{3}$ [42]. Estas dificuldades de processamento resultaram em três linhas de pesquisas, que compartilham o objetivo de diminuir a temperatura de sinterização da cerâmica de AlN. A primeira linha de pesquisa envolve o uso de aditivo de sinterização, que promove a densificação do AlN entre 1700 e $1800{ }^{\circ} \mathrm{C}$ [43-45]. A diminuição da temperatura foi atribuída ao aumento da difusividade no estado sólido [46] e à formação de fase líquida em baixas temperaturas $[43,46]$. Neste caso,o aditivo óxido reage com a camada de óxido $\left(\alpha-\mathrm{Al}_{2} \mathrm{O}_{3}\right)$ presente intrinsecamente na superfície do pó de AlN, formando fases de aluminato, as quais fundem em altas temperaturas [47, 48], promovendo a densificação pelos mecanismos de rearranjo de partículas e solução-difusão-precipitação da sinterização com fase líquida [48]. Além de diminuir a temperatura, o aditivo de sinterização também melhora a condutividade térmica $(\lambda)$ da cerâmica de AlN $[47,48]$. Este assunto será discutido na próxima seção. A segunda linha de pesquisa envolve o uso de pó ultrafino (nanométrico) de AlN com e sem aditivo de sinterização, que possibilitou a densificação entre 1550 e $1650^{\circ} \mathrm{C}$ [49-52]. Esta rota de processamento não teve prosseguimento porque a cerâmica de AlN exibiu baixos valores de $\lambda(\sim 70 \mathrm{~W} / \mathrm{mK})$. A terceira linha de pesquisa envolve a utilização de aditivo multicomponente de sinterização, que resultou em cerâmicas densas de AlN com valores moderados de $\lambda(150-180 \mathrm{~W} / \mathrm{mK})$ após sinterização entre 1600 e $1750{ }^{\circ} \mathrm{C}$ [53-57]. Nos próximos tópicos, apresentam-se as influências do uso ou não de aditivos de sinterização, bem como o efeito da atmosfera de sinterização no comportamento de densificação do AlN.

AlN sem aditivo de sinterização: o coeficiente de autodifusão do AlN pode ser aumentado em função do 
aumento do teor de oxigênio $(\mathrm{O})$ presente inerentemente na superfície do pó de partida [58]. Durante a sinterização, a impureza $\mathrm{O}$ entra em solução sólida na rede cristalina do AlN, ocupando a posição do N, o que resulta na formação de lacunas de $\mathrm{Al}$, as quais aumentam a difusividade de massa em altas temperaturas [59]. Há um teor mínimo de $\mathrm{O}$, ao redor de $2 \%$ em massa, que deve estar na superfície do pó, para densificar o AlN por prensagem a quente na temperatura de $1800{ }^{\circ} \mathrm{C}$ [59]. A densificação do AlN "puro" (sem aditivo de sinterização) ocorre por sinterização no estado sólido, pois a camada de óxido amorfo sobre as partículas de AlN, cuja espessura é da ordem de 40 a $120 \AA$, cristaliza-se em $\alpha-\mathrm{Al}_{2} \mathrm{O}_{3}$ durante o aquecimento, principalmente acima de $1200{ }^{\circ} \mathrm{C}$ [58]. Em seguida, o AlN reage com a camada de $\alpha-\mathrm{Al}_{2} \mathrm{O}_{3}$, formando na superfície da partícula a fase espinélio de oxinitreto de alumínio $\left(\gamma\right.$ - $\mathrm{AlON}, \mathrm{Al}_{23} \mathrm{O}_{27} \mathrm{~N}_{5}$ ) acima de $1650{ }^{\circ} \mathrm{C}$, que favorece a densificação devido ao aumento da concentração de lacunas - aumento do coeficiente de autodifusão. A formação de outras fases como o pseudopolitipo $27 \mathrm{R}\left(\mathrm{Al}_{9} \mathrm{O}_{3} \mathrm{~N}_{7}\right)$ acima de $1800{ }^{\circ} \mathrm{C}$ também pode auxiliar a densificação [58]. Contudo, outros estudos $[39,51]$ sugeriram que a densificação do AlN "puro" pode ser assistida por sinterização com fase líquida, já que o diagrama de fases $\mathrm{AlN}-\mathrm{Al}_{2} \mathrm{O}_{3}$ prevê a formação de fase líquida ao redor de $1850{ }^{\circ} \mathrm{C}$. Enfim, a qualidade do pó de AlN melhorou ao longo do tempo, sendo estabelecido que o comportamento de densificação do AlN é dependente das características do pó de partida, pois a utilização de pó fino com estreita distribuição granulométrica $(\leq 1$ $\mu \mathrm{m})$ e baixo teor de $\mathrm{O}(\sim 1 \%$ em massa) pode promover a densificação do AlN "puro" ( $\geq 90 \%$ da densidade teórica) por sinterização sem pressão a partir de $1900{ }^{\circ} \mathrm{C}$ [48].

AlN com aditivo de sinterização: os óxidos de ítrio $\left(\mathrm{Y}_{2} \mathrm{O}_{3}\right)$ e de cálcio $(\mathrm{CaO})$ são os aditivos de sinterização mais usados nos estudos de cerâmica de $\mathrm{AlN}$, mas o $\mathrm{Y}_{2} \mathrm{O}_{3}$ se tornou mais popular porque sua utilização resulta em cerâmicas com maiores valores de $\lambda$ [60-62]. A fabricação de $\mathrm{AlN} \operatorname{com~} \mathrm{Y}_{2} \mathrm{O}_{3}$ ou $\mathrm{CaO}$ está bem estabelecida, sendo obtidas cerâmicas com $\lambda$ variando de 140 a $200 \mathrm{~W} / \mathrm{mK}$ [44, 63, 64]. A cerâmica de AlN com $\mathrm{Y}_{2} \mathrm{O}_{3}$ requer temperaturas de sinterização ao redor de $1800{ }^{\circ} \mathrm{C}$ para atingir alta densidade [60-62]. Isto ocorre porque a densificação é assistida por fase líquida e as menores temperaturas eutéticas dos sistemas AlN$\mathrm{Al}_{2} \mathrm{O}_{3}-\mathrm{Y}_{2} \mathrm{O}_{3}$ e $\mathrm{Al}_{2} \mathrm{O}_{3}-\mathrm{Y}_{2} \mathrm{O}_{3}$ são previstas ao redor de 1720 e $1760{ }^{\circ} \mathrm{C}$, respectivamente [60]. Já os sistemas $\mathrm{AlN}-\mathrm{Al}_{2} \mathrm{O}_{3}-$ $\mathrm{CaO}$ e $\mathrm{Al}_{2} \mathrm{O}_{3}-\mathrm{CaO}$ apresentam temperaturas eutéticas ao redor de 1290 e $1390{ }^{\circ} \mathrm{C}$, respectivamente [60]. Estes resultados sugerem que a adição de compostos à base de cálcio pode densificar o AlN em menores temperaturas do que a adição de compostos à base de ítrio. Porém, o AlN com aditivo contendo cálcio atinge máxima densificação somente entre 1700 e $1800{ }^{\circ} \mathrm{C}[42,44,50,60]$, indicando que esta cerâmica requer elevadas temperaturas de sinterização em relação às suas baixas temperaturas eutéticas, ou seja, necessita de um superaquecimento de 200 a $300{ }^{\circ} \mathrm{C}$ acima da temperatura eutética mais baixa do sistema $\mathrm{CaO}-\mathrm{Al}_{2} \mathrm{O}_{3}[42,60]$. Assim sendo, verificouse que as cerâmicas de $\mathrm{AlN}$ com $\mathrm{Y}_{2} \mathrm{O}_{3}$ ou $\mathrm{CaO}$ requerem elevadas temperaturas de sinterização $\left(>1700{ }^{\circ} \mathrm{C}\right)$ para atingir máxima densificação, o que aumenta os custos de produção em comparação com os substratos de alumina (96\% de $\mathrm{Al}_{2} \mathrm{O}_{3}$ ), que são produzidos comercialmente entre 1500 e $1600{ }^{\circ} \mathrm{C}$ [8]. Apesar disso, há empresas (Sumitomo Electric USA, Toshiba Ceramics, Kyocera, AdTech Ceramics, Coorstek, CeramTec, Accuratus, Maruwa, Tokuyama) comercializando substratos de AlN $\operatorname{com} \lambda$ variando de 100 a $230 \mathrm{~W} / \mathrm{mK}$.

AlN com sistema multicomponente de aditivo de sinterização: a adição simultânea de compostos à base de ítrio e cálcio promove a densificação do AlN entre 1650 e $1750{ }^{\circ} \mathrm{C}$ [53-56], além de resultar em valores de $\lambda$ da ordem de $180 \mathrm{~W} / \mathrm{mK}[55,56]$. Já o sistema $\mathrm{Li}_{2} \mathrm{O}_{-} \mathrm{Y}_{2} \mathrm{O}_{3}$ $\mathrm{CaO}$ pode densificar o AlN a $1600{ }^{\circ} \mathrm{C}[53,54]$, sendo alcançados valores de $\lambda$ próximos de $170 \mathrm{~W} / \mathrm{mK}$ [53]. Recentemente, foi demonstrado que o sistema $\mathrm{CaZrO}_{3}$ $\mathrm{Y}_{2} \mathrm{O}_{3}$ promove a densificação do AlN a partir de $1550{ }^{\circ} \mathrm{C}$, sendo observadas cerâmicas com $\lambda$ de $156 \mathrm{~W} / \mathrm{mK}$ após sinterização em duplo estágio (temperatura inicial de $1600{ }^{\circ} \mathrm{C}$ seguido de patamar a $1400{ }^{\circ} \mathrm{C}$ ) [38]. Em estudos comparativos, observou-se que o $\mathrm{AlN}$ com $\mathrm{CaO}$ teve maior retração linear do que o AlN com $\mathrm{CaO}-\mathrm{Y}_{2} \mathrm{O}_{3}$ durante a sinterização abaixo de $1600{ }^{\circ} \mathrm{C}[54,56]$. A menor retração do AlN com $\mathrm{CaO}-\mathrm{Y}_{2} \mathrm{O}_{3}$ foi atribuída à adição de $\mathrm{Y}_{2} \mathrm{O}_{3}$, que influenciou na formação de compostos do sistema CaAl-O, pois parte do $\mathrm{Al}_{2} \mathrm{O}_{3}$ presente sobre as partículas de AlN foi consumida na formação de compostos do sistema Y-Al-O (fases mais refratárias) [56]. Deste modo, o AlN com $\mathrm{CaO}-\mathrm{Y}_{2} \mathrm{O}_{3}$ teve menor quantidade de fase líquida e, portanto, menor retração linear durante a sinterização abaixo de $1600{ }^{\circ} \mathrm{C}$. Por outro lado, o AlN com CaO- $\mathrm{Y}_{2} \mathrm{O}_{3}$ exibiu aumento significativo de retração linear durante os tratamentos isotérmicos a $1600{ }^{\circ} \mathrm{C}$, enquanto o AlN com $\mathrm{CaO}$ teve aumento inexpressivo de retração linear para as mesmas condições experimentais [56]. Em relação ao AlN com $\mathrm{CaO}$, sugeriu-se que a composição do líquido $\mathrm{Ca}$ Al-O mudou para composições mais refratárias durante a sinterização devido ao enriquecimento com $\mathrm{Al}_{2} \mathrm{O}_{3}$ contido no pó de $\mathrm{AlN}$, o que limitou a densificação na temperatura de $1600{ }^{\circ} \mathrm{C}$ [65]. Porém, quando $\mathrm{CaO}$ e $\mathrm{Y}_{2} \mathrm{O}_{3}$ foram adicionados concomitantemente ao AlN, a composição da fase líquida mudou para a região central do diagrama de fases do sistema $\mathrm{CaO}-\mathrm{Y}_{2} \mathrm{O}_{3}-\mathrm{Al}_{2} \mathrm{O}_{3}$, onde localizam-se as fases ternárias $\left(\mathrm{CaYAlO}_{4}\right.$ e $\left.\mathrm{CaYAl}_{3} \mathrm{O}_{7}\right)$ [65]. Quando estas fases ternárias são formadas, impede-se a formação de fases mais refratárias e modifica-se o comportamento de viscosidade da fase líquida, o que contribui para a densificação do AlN em baixas temperaturas [65]. A formação de fase líquida a partir de óxidos complexos do sistema Ca-Y-Al-O é prevista ao redor de $1600{ }^{\circ} \mathrm{C}$ [57]. Em relação aos sistemas $\mathrm{Li}_{2} \mathrm{O}-\mathrm{Y}_{2} \mathrm{O}_{3}-\mathrm{CaO}$ e $\mathrm{LiYO}_{2}-\mathrm{CaO}$, estabeleceu-se que o AlN densificou a $1600{ }^{\circ} \mathrm{C}$ porque os compostos à base de lítio melhoraram a molhabilidade da fase líquida, bem como diminuíram a temperatura eutética 
dos compostos dos sistemas $\mathrm{Al}_{2} \mathrm{O}_{3}$-óxidos metálicos [53]. Além disso, os compostos à base de lítio possuem elevada pressão de vapor, principalmente entre 1300 e $1600{ }^{\circ} \mathrm{C}$, o que promoveu uma significativa evaporação de compostos, aumentando $\lambda$ devido à diminuição da quantidade de segundas fases de contorno de grão [53]. Os resultados citados demostram que é possível obter cerâmicas densas de AlN com valores moderados de $\lambda(150-170 \mathrm{~W} / \mathrm{mK})$ após sinterização ao redor de $1600{ }^{\circ} \mathrm{C}$, o que possibilita adequar o AlN ao processo HTCC estabelecido para o $\mathrm{Al}_{2} \mathrm{O}_{3}$ entre 1500 e $1600{ }^{\circ} \mathrm{C}$ [8]. Apesar dos resultados promissores, observaram-se poucos estudos [53-57, 65, 66] sobre o comportamento de densificação do AlN com sistema multicomponente de aditivo de sinterização.

Efeito da atmosfera de sinterização: a cerâmica de AlN é comumente sinterizada em forno com elemento resistivo de grafita e sob atmosfera de gás nitrogênio. Deste modo, estabelece-se uma atmosfera de nitrogênio parcialmente redutora (com monóxido de carbono, $\mathrm{CO}$ ) durante a sinterização, que é considerada como um dos mecanismos responsáveis pela melhora da condutividade térmica $(\lambda)[63,67-72]$. A atmosfera redutora promove a desoxidação das segundas fases de contorno de grão, melhorando o $\lambda$ devido à evaporação de compostos da microestrutura $[16,63,68,69,73]$. Reações químicas, que descrevem o processo de desoxidação das segundas fases, foram propostas para o $\mathrm{AlN} \operatorname{com} \mathrm{Y}_{2} \mathrm{O}_{3}[68]$ :

$$
\begin{aligned}
& \mathrm{Al}_{2} \mathrm{O}_{3(\mathrm{~s})}+\mathrm{N}_{2(\mathrm{~g})}+3 \mathrm{CO}_{(\mathrm{g})} \leftrightarrow 2 \mathrm{AlN}_{(\mathrm{s})}+3 \mathrm{CO}_{2(\mathrm{~g})} \\
& \mathrm{Al}_{2} \mathrm{Y}_{4} \mathrm{O}_{9(\mathrm{~s})}+\mathrm{N}_{2(\mathrm{~g})}+3 \mathrm{CO}_{(\mathrm{g})} \leftrightarrow 2 \mathrm{AlN}_{(\mathrm{s})}+2 \mathrm{Y}_{2} \mathrm{O}_{3(\mathrm{~s})}+3 \mathrm{CO}_{2(\mathrm{~g})} \\
& \mathrm{Y}_{2} \mathrm{O}_{3(\mathrm{~s})}+\mathrm{N}_{2(\mathrm{~g})}+3 \mathrm{CO}_{(\mathrm{g})} \leftrightarrow 2 \mathrm{YN}_{(\mathrm{s})}+3 \mathrm{CO}_{2(\mathrm{~g})} \\
& \mathrm{CO}_{2(\mathrm{~g})}+\mathrm{C}_{(\mathrm{s})} \leftrightarrow 2 \mathrm{CO}_{(\mathrm{g})}
\end{aligned}
$$

Parte do gás $\mathrm{CO}_{2}$ produzido durante o processo de desoxidação das segundas fases (reações $\mathrm{D}$ a $\mathrm{F}$ ) pode ser convertida em gás $\mathrm{CO}$ (reação $\mathrm{G}$ ) devido à presença de $\mathrm{C}$ proveniente da grafita do cadinho e do elemento de aquecimento do forno. Deste modo, a atmosfera redutora pode ser mantida durante a sinterização. Embora haja consenso para utilizar atmosfera redutora na otimização de $\lambda$, a presença de uma atmosfera fortemente redutora pode inibir a densificação e, assim, diminuir drasticamente o $\lambda$ [16]. Há procedimentos frequentemente usados para impedir a formação de atmosfera excessivamente redutora, tais como: utilização de cadinho de nitreto de boro (BN) ou cadinho de grafita com camada protetora (“cama”) de pó de BN ou AlN [16]. Alguns estudos $[16,67]$ utilizaram pó de grafita ou de negro de fumo como aditivo de sinterização, objetivando a

\begin{tabular}{|c|c|c|c|}
\hline Condição de sinterização & Aditivo (\% massa) & $\lambda(\mathrm{W} / \mathrm{mK})$ & Ref. \\
\hline Forno de grafite; cadinho de $\mathrm{AlN}$; sinterização a $1850^{\circ} \mathrm{C}-100 \mathrm{~min}$ & $2 \% \mathrm{Y}_{2} \mathrm{O}_{3}$ & 151 & {$[47]$} \\
\hline Forno de grafite; cadinho de $\mathrm{AlN}$; sinterização a $1850^{\circ} \mathrm{C}-100 \mathrm{~min}$ & $2 \% \mathrm{Dy}_{2} \mathrm{O}_{3}$ & 160 & {$[47]$} \\
\hline Forno de grafite; cadinho de AlN; sinterização a $1850^{\circ} \mathrm{C}-100$ min & $2 \% \mathrm{MgO}$ & 63 & {$[47]$} \\
\hline Forno de grafite; cadin.ho de AlN; sinterização a $1850^{\circ} \mathrm{C}-100$ min & $2 \% \mathrm{BaO}$ & 83 & {$[47]$} \\
\hline $\begin{array}{l}\text { Forno de grafite; cadinho de AlN; cama de pó de AlN; sinterização a } \\
\qquad 1800^{\circ} \mathrm{C}-3 \mathrm{~h}\end{array}$ & $10 \% \mathrm{CaO}$ & 140 & {$[64]$} \\
\hline $\begin{array}{l}\text { Forno de grafite; cadinho de AlN; sinterização a } 1850^{\circ} \mathrm{C}-100 \mathrm{~min} \text {; } \\
\text { tratamento térmico de pós-sinterização a } 1850^{\circ} \mathrm{C}-1000 \mathrm{~min}\end{array}$ & $4,9 \% \mathrm{Y}_{2} \mathrm{O}_{3}$ & 232 & {$[80]$} \\
\hline $\begin{array}{l}\text { Forno de grafite; cadinho de AlN; sinterização a } 1850^{\circ} \mathrm{C} \text { - } \\
100 \mathrm{~min} \text {; tratamento térmico de pós-sinterização a } 1850^{\circ} \mathrm{C}-1000 \text { min }\end{array}$ & $7,9 \% \mathrm{Dy}_{2} \mathrm{O}_{3}$ & 205 & {$[80]$} \\
\hline $\begin{array}{l}\text { Forno de grafite; cadinho de AlN; sinterização a } 1850^{\circ} \mathrm{C}-100 \mathrm{~min} \text {; } \\
\text { tratamento térmico de pós-sinterização a } 1850^{\circ} \mathrm{C}-1000 \mathrm{~min}\end{array}$ & $7,3 \% \mathrm{Sm}_{2} \mathrm{O}_{3}$ & 220 & {$[80]$} \\
\hline $\begin{array}{l}\text { Forno de tungstênio; sinterização a } 1800^{\circ} \mathrm{C}-1 \mathrm{~h} \text {; tratamento térmico de } \\
\text { pós-sinterização em cadinho de grafite a } 1900{ }^{\circ} \mathrm{C}-100 \mathrm{~min}\end{array}$ & $5,2 \% \mathrm{Y}_{2} \mathrm{O}_{3}$ & 272 & {$[71]$} \\
\hline $\begin{array}{l}\text { Forno de tungstênio; cadinho de tungstênio; sinterização a } \\
1800^{\circ} \mathrm{C}-1 \mathrm{~h}\end{array}$ & $5 \% \mathrm{Y}_{2} \mathrm{O}_{3}+0,56 \% \mathrm{C}$ & 224 & {$[67]$} \\
\hline Cadinho de AlN; sinterização a $1600^{\circ} \mathrm{C}-6$ h & $\begin{array}{l}4 \% \mathrm{LiYO}_{2}+0,5 \% \\
\mathrm{CaO}\end{array}$ & 172 & {$[53]$} \\
\hline $\begin{array}{l}\text { Forno de grafite; cadinho de AlN; remoção de orgânicos a } 600{ }^{\circ} \mathrm{C} \text {; } \\
\text { sinterização a } 1650^{\circ} \mathrm{C}-6 \mathrm{~h}\end{array}$ & $4 \% \mathrm{YF}_{3}+2 \% \mathrm{CaF}_{2}$ & 187 & {$[55]$} \\
\hline $\begin{array}{l}\text { Forno de grafite; cadinho de } \mathrm{BN} \text {; sinterização em duplo estágio nas } \\
\text { temperaturas de } 1600^{\circ} \mathrm{C}-3 \mathrm{~h} \mathrm{e} 1400{ }^{\circ} \mathrm{C}-3 \mathrm{~h}\end{array}$ & $\begin{array}{c}3 \% \mathrm{CaZrO}_{3}+3 \% \\
\mathrm{Y}_{2} \mathrm{O}_{3} \\
\end{array}$ & 156 & [38] \\
\hline
\end{tabular}
formação in situ de uma atmosfera redutora dentro do corpo

Tabela III - Condutividade térmica $(\lambda)$ de cerâmicas de AlN preparadas com diferentes aditivos e condições de sinterização. [Table III - Thermal conductivity ( $\lambda$ ) of prepared AlN ceramics with different additives and sintering conditions.] 
conformado de AlN. A utilização de até $0,5 \%$ em massa de C aumentou consideravelmente o $\lambda$ do AlN "puro" em relação à cerâmica preparada sem $C$ [74]. Porém, adições de $C$ acima de $0,5 \%$ em massa causaram desoxidação excessiva, o que inibiu a densificação do AlN "puro" por prensagem a quente na temperatura de $1800{ }^{\circ} \mathrm{C}$ [74]. Em relação ao AlN com $\mathrm{Y}_{2} \mathrm{O}_{3}$, estabeleceu-se que a densificação acontece quando a razão $\mathrm{C} / \mathrm{O}$ é menor do que 1 [75]. O $\lambda$ aumenta em função do aumento da razão $\mathrm{C} / \mathrm{O}$, na faixa menor do que 1 , e quando se aproxima da condição limite para densificação $(\mathrm{C} / \mathrm{O}=1)$, as segundas fases apresentam tendência para formar fases mais refratárias ricas em $\mathrm{Y}_{2} \mathrm{O}_{3}$. Embora a composição do aluminato de ítrio dependa do teor de $\mathrm{Y}_{2} \mathrm{O}_{3}$ e do teor de O presente na superfície do pó de AlN [75, 76], durante a sinterização sob atmosfera redutora, a composição das segundas fases muda gradualmente de $\mathrm{xAl}_{2} \mathrm{O}_{3} \cdot \mathrm{yY}_{2} \mathrm{O}_{3}$ para $\mathrm{Y}_{2} \mathrm{O}_{3}$ devido à maior pressão parcial de oxigênio do $\mathrm{Al}_{2} \mathrm{O}_{3}$ $[67,76]$.

Em relação ao $\mathrm{AlN}$ com $\mathrm{CaO}$, a evaporação de compostos ocorre durante a sinterização tanto em atmosfera redutora $[48,64,77]$ como inerte (forno com elemento resistivo de tungstênio) [42]. Neste caso, observou-se maior evaporação de compostos à base de cálcio devido à elevada pressão de vapor do $\mathrm{CaO}$ [78]. Outros estudos [50,74] usaram os compostos $\mathrm{CaC}_{2}$ ou $\mathrm{CaCN}_{2}$ para formar in situ uma atmosfera redutora no interior do corpo de AlN. Após sinterização sob pressão a $1800{ }^{\circ} \mathrm{C}$, o AlN com $2 \%$ em massa de $\mathrm{CaC}_{2}$ atingiu um $\lambda$ de $180 \mathrm{~W} / \mathrm{mK}$, enquanto o AlN com $2 \%$ em massa de $\mathrm{CaO}$ teve um $\lambda$ próximo de $140 \mathrm{~W} / \mathrm{mK}$ [74]. Porém, o AlN com $2 \%$ em massa de $\mathrm{CaC}_{2}$ sinterizado sem pressão a $1800{ }^{\circ} \mathrm{C}$ apresentou um baixo valor de $\lambda(\sim 95 \mathrm{~W} / \mathrm{mK})$ [79]. Outra prática usual nos estudos sobre a otimização de $\lambda$ é remover (evaporar) as segundas fases de contorno de grão por meio de tratamento térmico pós-sinterização (annealing) sob atmosfera redutora em altas temperaturas $\left(>1800{ }^{\circ} \mathrm{C}\right)$ [63, 69-71, 80]. Porém, este procedimento induz crescimento de grão, que afeta drasticamente as propriedades mecânicas do produto final. Isto mostra que os estudos sobre a otimização de $\lambda$ não se preocuparam com a otimização de outras propriedades, como a resistência mecânica e a tenacidade à fratura, que são propriedades importantes para a tecnologia de componentes passivos, pois o substrato e o material para encapsulamento devem fornecer sustentação e integridade física ao chip. Em suma, as rotas de processamento empregadas para otimizar o $\lambda$ do AlN envolvem a melhora na qualidade do pó de partida, adição de aditivo de sinterização, utilização de atmosfera redutora e tratamento térmico pós-sinterização. A Tabela III mostra uma compilação de valores de $\lambda$ para o AIN preparado com diferentes aditivos e condições de sinterização.

\section{CONDUTIVIDADE TÉRMICA ( $\lambda$ ) DO NITRETO DE ALUMÍNIO}

A cerâmica policristalina de AlN sem aditivo de sinterização tem $\lambda$ variando de 50 a $95 \mathrm{~W} / \mathrm{mK}$ à temperatura ambiente [73, 74]. Estes valores são considerados baixos, visto que o monocristal de AlN isento de oxigênio $(\mathrm{O})$ pode alcançar valores de $\lambda$ ao redor de $320 \mathrm{~W} / \mathrm{mK}$ à temperatura ambiente [15]. Um dos fatores que afetam o $\lambda$ é a entrada de $\mathrm{O}$ em solução sólida na rede cristalina do AlN durante a sinterização, pois a substituição de $\mathrm{N}$ por $\mathrm{O}$ na rede cristalina leva à formação de lacunas de $\mathrm{Al}$, as quais diminuem o livre caminho médio de propagação dos fônons (portadores de calor) devido ao espalhamento causado pela interação lacuna-fônon [15, 73, 81]. Esta impureza, O, é oriunda da camada de óxido presente intrinsecamente na superfície do pó de AlN. Quanto maior o teor de O no pó de AlN, maior é a diminuição de $\lambda$ [81]. Além das lacunas de $\mathrm{Al}$, o $\mathrm{O}$ em solução sólida induz a formação de diferentes tipos de defeitos cristalinos (por ex., falhas de empilhamento e discordâncias), que também causam a diminuição do livre caminho médio dos fônons $[82,83]$. $\lambda$ também é influenciado pela temperatura, pois o $\lambda$ do monocristal de AlN diminuiu de 320 para $100 \mathrm{~W} / \mathrm{mK}$ com o aumento da temperatura de 27 para $327^{\circ} \mathrm{C}$ [15]. Apesar disso, o referido valor de $\lambda$ do AlN $\left(100 \mathrm{~W} / \mathrm{mK}\right.$ a $\left.327^{\circ} \mathrm{C}\right)$ é cerca de 5 vezes maior do que o $\lambda$ do $\mathrm{Al}_{2} \mathrm{O}_{3}$ à temperatura ambiente (20-30 $\mathrm{W} / \mathrm{mK}, 25^{\circ} \mathrm{C}$ ). $\mathrm{O} \lambda$ do AlN policristalino pode ser otimizado por meio do uso de aditivo de sinterização, pois o aditivo óxido reage com a camada de óxido sobre o pó de AlN, formando segundas fases de contorno de grão, o que evita a formação de solução sólida de $\mathrm{O}$ e, com isso, $\lambda$ aumenta devido à diminuição de defeitos cristalinos (por ex., lacunas de $\mathrm{Al}$, falhas de empilhamento e discordâncias) [47, 48]. Entretanto, há relatos $[43,84]$ indicando que alguns óxidos metálicos podem promover a oxidação do AlN durante a sinterização, o que resultaria no aumento da quantidade de $\mathrm{O}$ e consequente redução de $\lambda$.

A Fig. 1 mostra uma compilação de valores de $\lambda \mathrm{em}$ função do teor de aditivo de sinterização para a cerâmica de AlN. O aumento de $\lambda$ em função do teor de $\mathrm{Y}_{2} \mathrm{O}_{3}$ se deve ao fato de que, para adições abaixo de $5 \%$ em massa, a quantidade de aditivo foi insuficiente para reagir com todo O presente na superfície do pó de AlN. Deste modo, o O residual, que não reagiu com o $\mathrm{Y}_{2} \mathrm{O}_{3}$, entrou em solução sólida no AlN, limitando a otimização de $\lambda$. Para adições entorno de $5 \%$ em massa, todo $\mathrm{O}$ foi consumido na reação com o $\mathrm{Y}_{2} \mathrm{O}_{3}$, o que evitou a formação de solução sólida de $\mathrm{O}$ no AlN, resultando em máximos valores de $\lambda$. Assim sendo, adições acima de $5 \%$ em massa de $\mathrm{Y}_{2} \mathrm{O}_{3}$ apenas contribuíram para aumentar a quantidade de segundas fases de contorno de grão. Isto levou ao completo molhamento dos contornos de grão pelas segundas fases, o que diminuiu o contato entre os grãos de AlN, causando a diminuição de $\lambda$, pois as segundas fases apresentam baixos valores de $\lambda[38,47,72]$. Tratamento térmico pós-sinterização em atmosfera redutora tem sido usado para evaporar as segundas fases, o que promove a "limpeza" dos contornos de grão, maximizando os valores de $\lambda$ ( $>200 \mathrm{~W} / \mathrm{mK}$, Tabela III). Sabendo que este procedimento causa significativo crescimento dos grãos de AlN [47], pode-se dizer que $\lambda$ também aumentou devido à diminuição da quantidade de contornos de grão (regiões de defeito) na microestrutura. Deste modo, pode-se dizer 
que os moderados valores de $\lambda(150-180 \mathrm{~W} / \mathrm{mK})$, obtidos em cerâmicas de AlN sinterizadas em baixas temperaturas $\left(\leq 1650{ }^{\circ} \mathrm{C}\right)$, são decorrentes das microestruturas com grãos finos, que conferem maiores frações de zonas defeituosas (contornos de grão). Em relação à adição de $\mathrm{CaO}, \lambda$ mostrou uma tendência de aumento linear em função do aumento do teor de aditivo (Fig. 1). Isto ocorreu porque o $\mathrm{CaO}$ tem elevada pressão de vapor [78], o que induziu elevada evaporação de compostos em temperaturas tão baixas quanto $1100{ }^{\circ} \mathrm{C}$ [42], sendo necessário aumentar o teor de $\mathrm{CaO}$ para ter aditivo suficiente para reagir com o $\mathrm{Al}_{2} \mathrm{O}_{3}$ contido no pó de $\mathrm{AlN}$. Embora o $\mathrm{CaO}$ promova evaporação intrínseca de segundas fases durante a sinterização, os valores de $\lambda$ obtidos com $\mathrm{CaO}$ não foram superiores àqueles alcançados com $\mathrm{Y}_{2} \mathrm{O}_{3}$ (Fig. 1). Uma explicação para este fenômeno é que a densidade do AlN diminui em função do aumento do teor de $\mathrm{CaO}[42,38,65,77]$, o que indica a formação de poros, os quais limitam a condução de calor pela microestrutura. Em suma, a cerâmica de AlN tem extensa faixa de valores de $\lambda(50$ a $270 \mathrm{~W} / \mathrm{mK})$ devido às diferenças nas características do pó de partida, aos diferentes aditivos de sinterização, aos diferentes parâmetros de sinterização e ao tratamento térmico pós-sinterização sob atmosfera redutora.

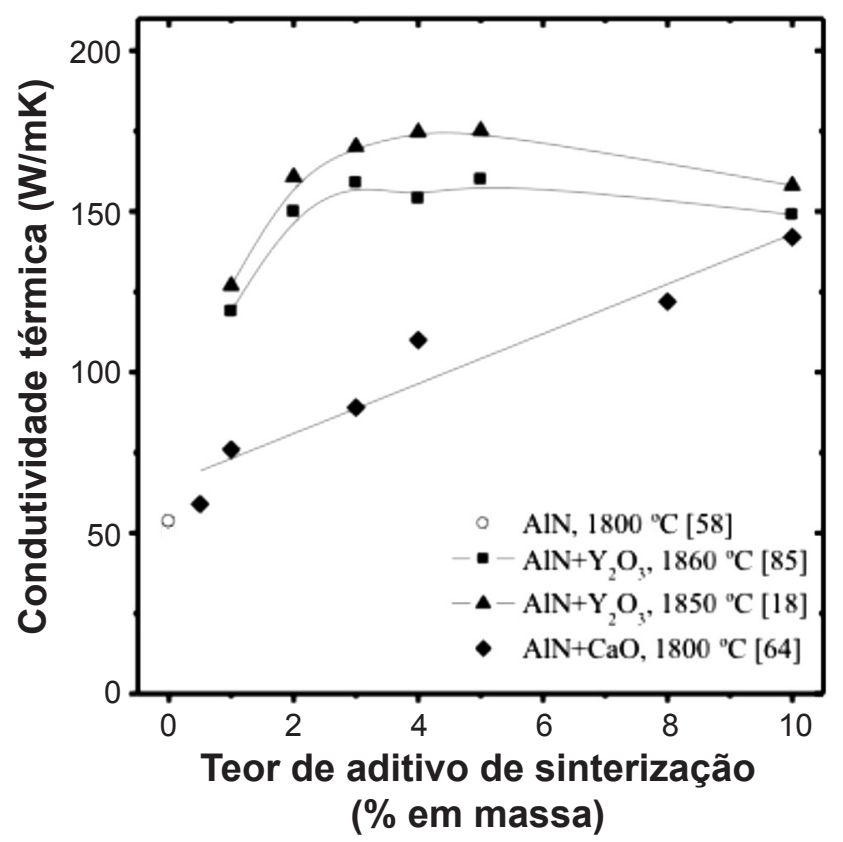

Figura 1: Condutividade térmica $(\lambda)$ em função do teor de aditivo para cerâmicas de AlN sinterizadas nas temperaturas indicadas.

[Figure 1: Thermal conductivity ( $\lambda$ ) as a function of additive content for AlN ceramics sintered at the indicated temperatures.]

\section{PROPRIEDADES MECÂNICAS DO NITRETO DE ALUMÍNIO}

Os módulos de potência, principalmente os fabricados pela tecnologia $\mathrm{DBC}$, trabalham em uma extensa faixa de temperatura de -55 a $+200{ }^{\circ} \mathrm{C}$ [86]. Estes dispositivos eletrônicos são susceptíveis à falha por fadiga térmica porque utilizam materiais (por ex., $\mathrm{Cu}, \mathrm{AlN}$ e $\mathrm{Si}$ ) com diferentes coeficientes de expansão térmica [38, 86, 87]. Outros dispositivos eletrônicos, como os lasers de alta energia, são susceptíveis à falha por choque térmico porque apresentam problemas de gerenciamento térmico, sendo esta falha minimizada pela utilização de materiais com boas propriedades mecânicas e térmicas [88]. Embora haja interesse por materiais com boa confiabilidade mecânica para a fabricação de dispositivos eletrônicos de alta potência, os estudos sobre a cerâmica de AlN, por décadas, limitaram-se apenas a otimização de $\lambda$, postergando ou desconsiderando a otimização de outras propriedades, que também são relevantes para a fabricação destes dispositivos. Além da aplicação em microeletrônica, a cerâmica de AlN foi cogitada como material estrutural para aplicações em baixa e alta temperatura [8991], mas o estudo sobre o comportamento mecânico do AlN é limitado em comparação com outras cerâmicas estruturais $\left(\mathrm{Al}_{2} \mathrm{O}_{3}, \mathrm{SiC}\right.$ e $\left.\mathrm{Si}_{3} \mathrm{~N}_{4}\right)$ [92]. Há controvérsias em relação à aplicação do AlN como material estrutural, sendo observados os seguintes relatos: i) o AlN tem limitada aplicação devido à sua inerente fragilidade, bem como baixa resistência mecânica comparada com outras cerâmicas estruturais $\left(\mathrm{Si}_{3} \mathrm{~N}_{4}\right.$ e $\left.\mathrm{ZrO}_{2}\right)$ [93]; ii) apesar das boas propriedades mecânicas, o AlN tem limitada aplicação devido ao alto custo de produção [14]. Nos próximos tópicos, apresentam-se as propriedades mecânicas mais estudadas em relação às cerâmicas de $\mathrm{AlN}$ sem e com aditivo de sinterização.

\section{Resistência à flexão}

A Fig. 2 mostra uma compilação de valores de resistência à flexão $\left(\sigma_{\mathrm{f}}\right)$ em função da porosidade para as cerâmicas de AlN. $\mathrm{O} \sigma_{\mathrm{f}}$ do AlN sem aditivo diminuiu de 489 para 245 $\mathrm{MPa}$ em função do aumento da porosidade. Embora o $\sigma_{\mathrm{f}}$ do AlN seja influenciado pela porosidade, o comportamento mecânico do AlN também é influenciado pelo teor de O presente na superfície do pó de partida. Há um teor de O considerado "ótimo", pois o aumento do teor de O, até ao redor de 2,7\% em massa, promoveu um aumento significativo no $\sigma_{\mathrm{f}}$ do AlN ( $\left.\sim 500 \mathrm{MPa}\right)$ [102]. Este aumento de $\sigma_{\mathrm{f}}$ está associado ao aumento de densidade, já que é necessário um teor mínimo de $\mathrm{O}$, ao redor de $2 \%$ em massa, na superfície do pó de AlN para promover sua densificação por prensagem a quente a $1800{ }^{\circ} \mathrm{C}$ [59]. Acima de $2,7 \% \mathrm{em}$ massa de $\mathrm{O}, \mathrm{o} \sigma_{\mathrm{f}}$ diminuiu significativamente, atingindo valores menores do que $300 \mathrm{MPa}$ para o AlN com $12 \%$ em massa de $\mathrm{O}$. A diminuição de $\sigma_{\mathrm{f}}$ foi atribuída à formação dos politipóides 27R $\left(\mathrm{Al}_{9} \mathrm{O}_{3} \mathrm{~N}_{7}\right)$ e $12 \mathrm{H}\left(\mathrm{Al}_{6} \mathrm{O}_{3} \mathrm{~N}_{4}\right)$ [102]. Outro fator que influencia $\sigma_{\mathrm{f}}$ é a formação de solução sólida de $\mathrm{O}$ no $\mathrm{AlN}$, o que leva à formação de defeitos cristalinos, os quais enfraquecem os grãos de AlN e induzem o modo de fratura transgranular [99].

Embora $\sigma_{f}$ seja dependente da porosidade [103], os valores compilados de $\sigma_{\mathrm{f}}$ para o AIN com aditivo não foram consistentes (Fig. 2), como observado para o AlN sem aditivo. Em relação ao AlN com aditivo, os maiores valores de $\sigma_{\mathrm{f}}$ foram obtidos para o AlN com $\mathrm{CaZrO}_{3}-\mathrm{Y}_{2} \mathrm{O}_{3}(462-$ 


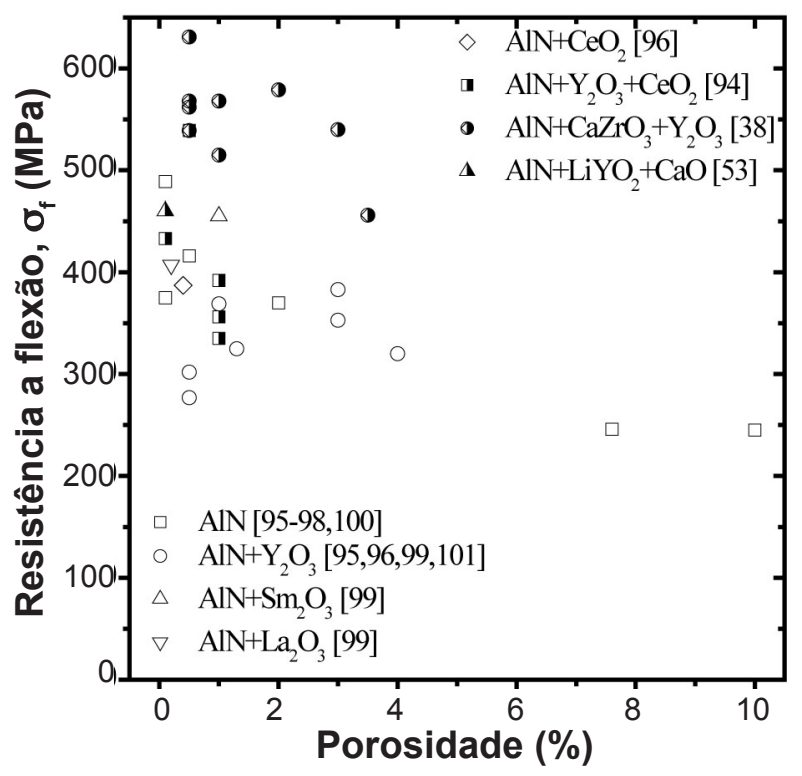

Figura 2: Resistência à flexão $\left(\sigma_{\mathrm{f}}\right)$ em função da porosidade para cerâmicas de AlN sem ou com 5\% em massa de aditivos de sinterização.

[Figure 2: Flexural strength $(\sigma)$ as a function of porosity for AlN ceramics without or with $\sim 5 \mathrm{wt} \%$ sintering aids.]

$631 \mathrm{MPa}$, Fig. 2). Neste sistema de aditivo, o $\sigma_{\mathrm{f}}$ diminuiu com o aumento da temperatura de sinterização, sendo este efeito associado ao crescimento de grão do $\mathrm{AlN}$, que aumentou de $\sim 1$ para $\sim 3 \mu \mathrm{m}$ entre 1500 e $1700{ }^{\circ} \mathrm{C}$. O sistema $\mathrm{LiYO}_{2}-\mathrm{CaO}$ densificou o AlN a $1600{ }^{\circ} \mathrm{C}$, o que conferiu um elevado valor de $\sigma_{\mathrm{f}}(460 \mathrm{MPa}$, Fig. 2) devido à formação de uma microestrutura homogênea com tamanho de grão ao redor de $2 \mu \mathrm{m}$. Para o sistema $\mathrm{Y}_{2} \mathrm{O}_{3}-\mathrm{CeO}_{2}, \mathrm{o} \sigma_{\mathrm{f}}$ diminuiu de 433 para $311 \mathrm{MPa}$ devido ao crescimento de grão do AlN, que aumentou de $\sim 2$ para 7,6 $\mu \mathrm{m}$ entre 1600 e 1800 ${ }^{\circ} \mathrm{C}$ (Fig. 2). Estes resultados mostraram que o $\sigma_{\mathrm{f}}$ pode ser otimizado pela diminuição do tamanho de grão do AlN, que é decorrente da diminuição da temperatura de sinterização por meio do uso de sistemas multicomponentes de aditivos de sinterização. Em contraste, o $\lambda$ do AlN é diminuído em função da diminuição do tamanho de grão [38]. O AlN com $\mathrm{Y}_{2} \mathrm{O}_{3}$ apresentou uma larga faixa de valores de $\sigma_{\mathrm{f}}(277$ a 383 MPa, Fig. 2), sendo este resultado atribuído às diferenças microestruturais (porosidade e tamanho de grão) causadas pelas diversas rotas de processamento, bem como pelos diferentes métodos de determinação de $\sigma_{\mathrm{f}}$. Em relação aos sistemas multicomponentes, o $\mathrm{Y}_{2} \mathrm{O}_{3}$ promoveu menores valores de $\sigma_{\mathrm{f}}$ porque necessita de maiores temperaturas de sinterização $\left(\geq 1800^{\circ} \mathrm{C}\right)$, o que induziu maior crescimento de grão. Os demais sistemas de aditivo $\left(\mathrm{Sm}_{2} \mathrm{O}_{3}, \mathrm{La}_{2} \mathrm{O}_{3}\right.$ e $\left.\mathrm{CeO}_{2}\right)$ também necessitam de elevadas temperaturas de sinterização, o que conferiu, de modo geral, menores valores de $\sigma_{\mathrm{f}} \mathrm{em}$ relação aos sistemas multicomponentes (Fig. 2).

Outro fator importante é que o aditivo de sinterização modifica o modo de fratura do AlN de transgranular para intergranular [95, 99, 104-106]. O predomínio de fratura intergranular foi atribuído, em primeiro lugar, ao aumento da resistência do grão de AlN, pois o aditivo óxido captura a impureza $\mathrm{O}$ da superfície do pó de AlN na forma de segundas fases, o que evita a formação de solução sólida de $\mathrm{O}$, reduzindo os defeitos cristalinos nos grãos de AlN [99]. Em segundo lugar, atribuiu-se à formação de tensões residuais nas regiões de contorno de grão devido às diferenças de expansão térmica entre o AlN e as segundas fases [88, 95, 104, 105]. Estas tensões residuais mudam de compressão para tração em função do aumento do teor de aditivo de sinterização [95]. Neste caso, estabeleceu-se que as segundas fases se concentram, preferencialmente, nas junções de três grãos (ou ponto triplo) para adições de até $2 \%$ em massa de $\mathrm{Y}_{2} \mathrm{O}_{3}, \mathrm{O}$ que resulta na formação de tensões residuais de compressão. $\mathrm{O}$ aumento do teor de $\mathrm{Y}_{2} \mathrm{O}_{3}$, acima de $2 \%$ em massa, faz com que as segundas fases se espalhem ao longo dos contornos de grão, o que leva à formação de tensões residuais de tração. A presença de tensões residuais de tração na interface AlN/ segundas fases enfraquece a ligação interfacial entre os grãos de AlN, favorecendo a propagação da trinca pelo contorno de grão [95]. Por exemplo, pequenas adições de $\mathrm{CaCO}_{3}(1 \%$ em massa) aumentaram o $\sigma_{\mathrm{f}}$, em relação ao AlN “puro", de 264 para $358 \mathrm{MPa}$ e, então, o $\sigma_{\mathrm{f}}$ diminuiu com o aumento do teor de aditivo (298 MPa para o AlN com $10 \%$ de $\mathrm{CaCO}_{3}$ ) [43]. Estes resultados indicaram que pequenas adições de aditivo podem aumentar a resistência mecânica do AlN porque minimizam os defeitos cristalinos nos grãos de AlN, assim como induzem tensões residuais de compressão nas regiões de contorno de grão.

A adição de $\mathrm{La}_{2} \mathrm{O}_{3}$ ou $\mathrm{Sm}_{2} \mathrm{O}_{3}$, em substituição ao $\mathrm{Y}_{2} \mathrm{O}_{3}$, aumentou o $\sigma_{\mathrm{f}}$ da cerâmica de AlN (Fig. 2). Neste caso, determinou-se que a adição de $\mathrm{La}_{2} \mathrm{O}_{3}$ ou $\mathrm{Sm}_{2} \mathrm{O}_{3}$ resultou em maior fração de fratura transgranular do que o $\mathrm{Y}_{2} \mathrm{O}_{3}$, o que levou à conclusão de que os dois óxidos $\left(\mathrm{La}_{2} \mathrm{O}_{3} \mathrm{e}\right.$ $\left.\mathrm{Sm}_{2} \mathrm{O}_{3}\right)$ aumentaram a tenacidade à fratura $\left(\mathrm{K}_{\mathrm{Ic}}\right)$ nas regiões de contorno de grão [99]. Neste caso, admitindo-se o mecanismo proposto em [95], pode-se dizer que as adições de $\mathrm{La}_{2} \mathrm{O}_{3}$ e $\mathrm{Sm}_{2} \mathrm{O}_{3}$ induziram menores tensões residuais de tração do que a adição de $\mathrm{Y}_{2} \mathrm{O}_{3}$ nas regiões de contorno de grão, o que resultou em menores frações de fratura intergranular e, consequentemente, em maiores valores de resistência mecânica. Contudo, a adição de $\mathrm{Sm}_{2} \mathrm{O}_{3}$ causou menor crescimento de grão $(3,3 \mu \mathrm{m})$ do que o $\mathrm{Y}_{2} \mathrm{O}_{3}(4 \mu \mathrm{m})$ e $\mathrm{La}_{2} \mathrm{O}_{3}(4 \mu \mathrm{m})$, o que deve ter contribuído para aumentar o $\sigma_{\mathrm{f}}$ do AlN. Comparativamente, observou-se que as cerâmicas de AlN (sem e com aditivo de sinterização) exibem valores de $\sigma_{\mathrm{f}}$ entre 290 e $631 \mathrm{MPa}$ (Fig. 2), enquanto as cerâmicas de $\mathrm{Al}_{2} \mathrm{O}_{3}$ têm valores de $\sigma_{\mathrm{f}}$ entre 280 e $980 \mathrm{MPa}[103,107,108]$. Estes resultados mostram que as cerâmicas de $\mathrm{AlN}$ e $\mathrm{Al}_{2} \mathrm{O}_{3}$ podem apresentar valores próximos de $\sigma_{\mathrm{f}}$, mas o $\mathrm{Al}_{2} \mathrm{O}_{3}$ pode alcançar valores significativamente maiores - cerca de 350 $\mathrm{MPa}$ acima do limite superior de $\sigma_{\mathrm{f}}$ do AlN.

\section{Tenacidade à fratura}

Em relação ao AlN sem aditivo, observou-se que o aumento da concentração de $\mathrm{O}$ no pó de partida induziu a formação de fases politipóides do sistema $\mathrm{AlN}-\mathrm{Al}_{2} \mathrm{O}_{3}$, as 
quais podem exibir uma morfologia de grãos alongados [109]. Porém, a presença de grãos alongados não ativou o mecanismo de tenacificação por deflexão de trinca, pois a propagação da trinca ocorreu pelo modo de fratura transgranular [110]. De fato, o AlN sem aditivo exibiu um comportamento de curva $\mathrm{R}$ (resistance curve) pouco significativo [110], sendo sugerido que os grãos de AlN foram enfraquecidos pela ocorrência de solução sólida de $\mathrm{O}$, o que favoreceu o modo de fratura transgranular $[52,99]$. Por outro lado, o AlN com 5\% em massa de $\mathrm{Y}_{2} \mathrm{O}_{3}$ exibiu um comportamento de curva $\mathrm{R}$, que foi atribuído à presença de tensões residuais nas regiões de contorno de grão [101]. Neste caso, concluiu-se que a tenacificação ocorreu pela ativação dos mecanismos de engastamento (interlocking) mecânico e/ou escorregamento com atrito das superfícies de fratura na zona de processo da cauda da trinca. Para o AlN com $2 \%$ em massa de $\mathrm{CaO}$, a tenacidade à fratura $\left(\mathrm{K}_{\mathrm{IC}}\right)$ aumentou em função do crescimento de grão (3 para $20 \mu \mathrm{m}$ ), sendo sugerida a ocorrência de tenacificação por deflexão de trica, com propagação da trinca pelo contorno de grão [104]. Em compósitos de SiC-AlN, indicou-se que o mecanismo de deflexão de trinca ocorreu devido à presença de tensões residuais geradas por diferenças tanto no módulo de elasticidade como no coeficiente de expansão térmica entre as duas fases [111].

Foi reportado que a diminuição da temperatura de 293 $\mathrm{K}$ para $77 \mathrm{~K}$ causou aumento significativo tanto de $\sigma_{\mathrm{f}}(364$ para $415 \mathrm{MPa})$ como de $\mathrm{K}_{\mathrm{Ic}}(3,98$ para 4,59 $\mathrm{MPa})$ para o AlN com $5 \%$ em massa de $\mathrm{Y}_{2} \mathrm{O}_{3}[106,107]$. Neste caso, a fração de fratura transgranular aumentou significativamente com a redução de temperatura, o que resultou no aumento de $\mathrm{K}_{\mathrm{Ic}}$ porque a propagação da trinca através do grão consome mais energia do que pelo contorno de grão [107]. Em relação ao mecanismo proposto em [95], pode-se dizer que a diminuição de temperatura, abaixo da temperatura ambiente, levou à diminuição da intensidade das tensões residuais nas regiões de contorno de grão, o que modificou o modo de fratura de intergranular para transgranular, aumentando os valores de $\sigma_{\mathrm{f}}$ e $\mathrm{K}_{\mathrm{Ic}}$. Comparativamente, observou-se que as cerâmicas de AlN, independente do uso ou não de aditivo, possuem valores de $\mathrm{K}_{\mathrm{Ic}}$ entre 2,9 e 4,6 $\mathrm{MPa} \cdot \mathrm{m}^{1 / 2}$ [93-95, $99,104,106,108,112]$, enquanto as cerâmicas de $\mathrm{Al}_{2} \mathrm{O}_{3}$ apresentam valores de $\mathrm{K}_{\mathrm{Ic}}$ entre 3,5 e 4,0 MPa.m ${ }^{1 / 2}[103$, 113]. Estes resultados mostraram que as cerâmicas de AlN e $\mathrm{Al}_{2} \mathrm{O}_{3}$ têm valores próximos de $\mathrm{K}_{\mathrm{Ic}}$, mas o $\mathrm{Al}_{2} \mathrm{O}_{3}$ pode ter maior resistência mecânica.

\section{Módulo de Young e dureza Vickers}

A Fig. 3 mostra uma compilação de valores de módulo de Young (E) em função da porosidade para cerâmicas de AlN sem e com aditivo de sinterização. $\mathrm{O}$ valor de $\mathrm{E}$ foi influenciado pela porosidade, mas não foi afetado pelos diferentes tipos de aditivos de sinterização (2\% em massa de $\mathrm{CaCO}_{3}, \mathrm{CaO}$ e $\mathrm{Y}_{2} \mathrm{O}_{3}$ ). Este efeito está detalhado em [90]. De modo geral, as cerâmicas "densas" de AlN (sem e com aditivo de sinterização) apresentam valores de E entre 300 e $335 \mathrm{MPa}[14,90,92,93,97,110,114-117]$, enquanto as cerâmicas "densas" de $\mathrm{Al}_{2} \mathrm{O}_{3}$ possuem valores de E entre 400 e $420 \mathrm{MPa}[14,103,107,108]$. Estes resultados mostraram que as cerâmicas de AlN apresentam rigidez menor do que as cerâmicas de $\mathrm{Al}_{2} \mathrm{O}_{3}$.

A Fig. 4 mostra uma compilação de valores de dureza

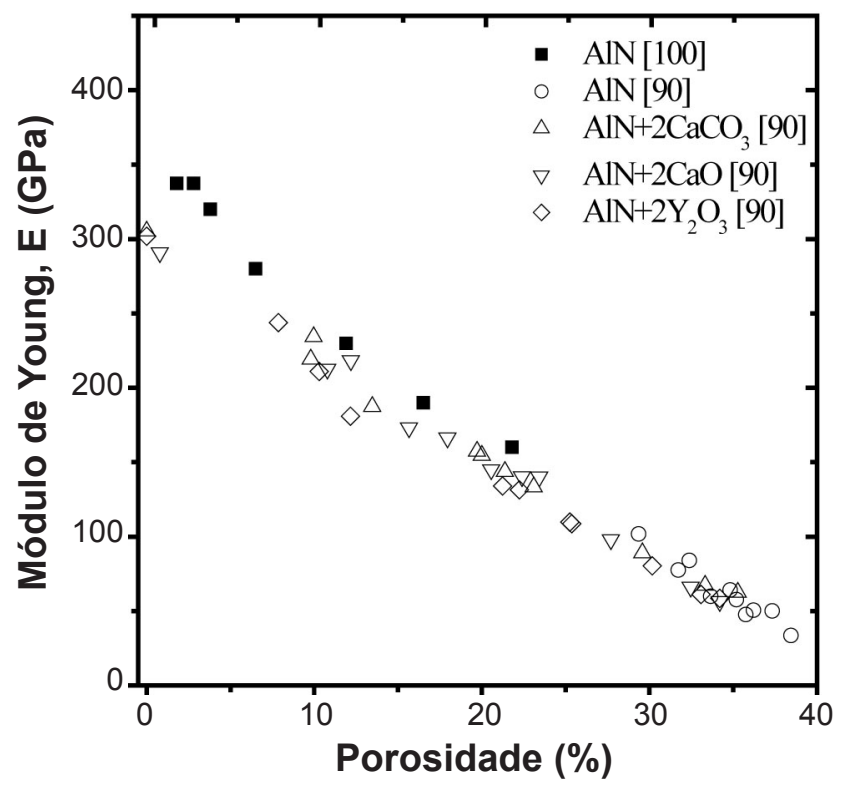

Figura 3: Módulo de Young (E) em função da porosidade para cerâmicas de AlN sem ou com $2 \%$ em massa de aditivo de sinterização.

[Figure 3: Young modulus (E) as a function of porosity for AlN ceramics without or with 2 wt\% sintering aid.]

Vickers $\left(\mathrm{H}_{\mathrm{v}}\right)$ em função da porosidade para as cerâmicas de AlN sem e com aditivo de sinterização. $\mathrm{O} \mathrm{H}_{\mathrm{v}}$ diminuiu em função do aumento da porosidade, tendo um comportamento similar àquele observado para $\sigma_{\mathrm{f}}$ (Fig. 2) e $\mathrm{E}$ (Fig. 3). Além da porosidade, $\mathrm{H}_{\mathrm{v}}$ foi influenciado pelo aumento do teor de $\mathrm{Y}_{2} \mathrm{O}_{3}$, pois a dureza diminuiu de 15,4 para $12 \mathrm{GPa}$ com o aumento de $2 \%$ para $6 \%$ em massa de aditivo de sinterização [95]. Outro fator importante é que a adição de $\mathrm{Y}_{2} \mathrm{O}_{3}$ resultou em cerâmicas de AlN mais duras do que a adição de $\mathrm{CaO}$ (Fig. 4), comparando-se cerâmicas processadas com a mesma quantidade de aditivo ( $2 \%$ em massa). Na literatura são reportadas cerâmicas de AlN (sem e com aditivo de sinterização) com valores de $\mathrm{H}_{\mathrm{v}}$ entre 5,9 e $16,4 \mathrm{GPa}[93$, 94, 97, 100, 104, 114], enquanto as cerâmicas de $\mathrm{Al}_{2} \mathrm{O}_{3}$ apresentam valores de $\mathrm{H}_{\mathrm{v}}$ entre 19,8 e $29 \mathrm{GPa}[103,107]$. Embora o AlN tenha menor dureza do que o $\mathrm{Al}_{2} \mathrm{O}_{3}$, este fato é visto como uma vantagem para o AlN, pois favorece o processo de usinagem durante a fabricação de dispositivos eletrônicos [2].

Em suma, as comparações entre as cerâmicas de $\mathrm{AlN}$ e $\mathrm{Al}_{2} \mathrm{O}_{3}$ mostraram que as duas cerâmicas possuem propriedades mecânicas $\left(\sigma_{\mathrm{f}}, \mathrm{K}_{\mathrm{Ic}}\right.$, E e $\left.\mathrm{H}_{\mathrm{V}}\right)$ relativamente próximas. Porém, dependendo da composição química do $\mathrm{Al}_{2} \mathrm{O}_{3}$, as propriedades $\sigma_{\mathrm{f}}$, E e $\mathrm{H}_{\mathrm{V}}$ do AlN tornamse significativamente menores. Além disso, o AlN tem propriedades mecânicas inferiores àquelas observadas para 


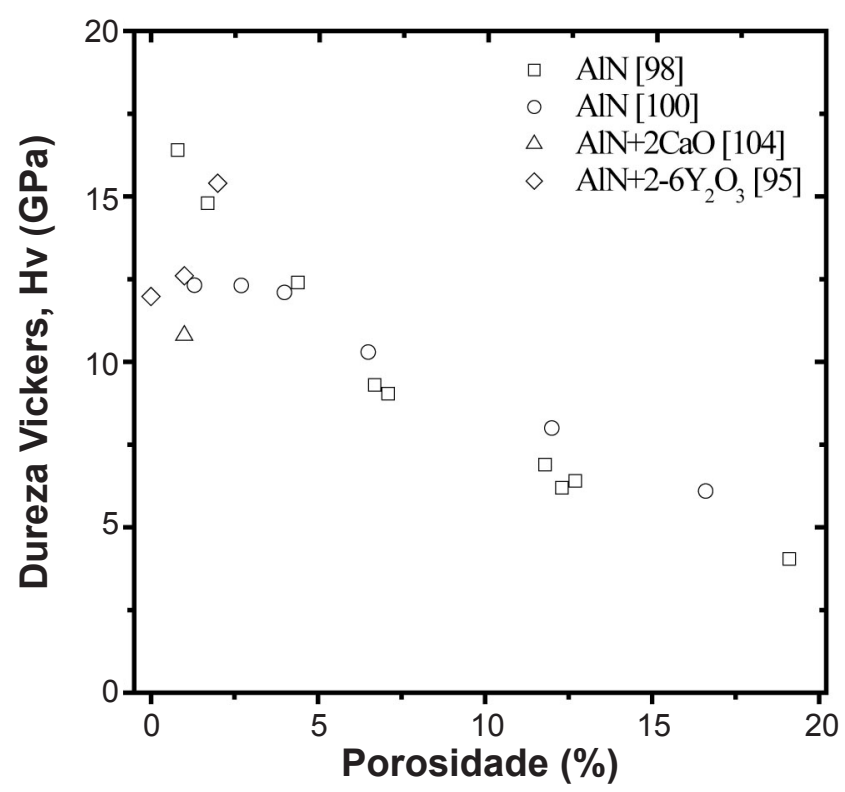

Figura 4: Dureza Vickers $\left(\mathrm{H}_{\mathrm{v}}\right)$ em função da porosidade para cerâmicas de AlN sem ou com 2-6\% em massa de aditivo de sinterização.

[Figure 4: Vickers hardness $\left(H_{V}\right)$ as a function of porosity for AlN ceramics without or with 2-6 wt\% sintering aid.]

as cerâmicas de $\mathrm{ZrO}_{2}, \mathrm{Si}_{3} \mathrm{~N}_{4}$ e $\mathrm{SiC}$ e, portanto, não oferece nenhum atrativo para aplicações estruturais devido ao seu elevado custo de produção em relação às cerâmicas óxidas. Esta explanação está de acordo com Faiote et al. [14], que destacaram as boas propriedades mecânicas do AlN, mas salientaram que seu elevado custo de produção limita sua aplicação como material estrutural. Entretanto, o AlN é considerado um potencial material para aplicações em proteção balística [91, 116]. Além de apresentar um comportamento de fratura com transição dúctil-frágil [91, 116], o AlN ainda apresenta uma mudança no modo de fratura em função da variação da taxa de deformação [116], que são comportamentos mecânicos desejáveis em aplicações balísticas. Este fato pode contribuir para a inclusão do AlN no mercado de materiais cerâmicos estruturais.

\section{RESISTÊNCIA À CORROSÃO DO NITRETO DE ALUMÍNIO}

Outra propriedade de interesse para a indústria de microeletrônica é a resistência à corrosão em soluções aquosas com pH ácido ou alcalino, pois os componentes cerâmicos para encapsulamento encontram várias etapas tanto de metalização como de lavagem nestas soluções antes dos processos de soldagem e selagem hermética [117]. O substrato cerâmico de AlN é severamente corroído em soluções alcalinas, mas sua taxa de corrosão foi diminuída pela formação de uma camada de proteção de oxinitreto de alumínio via oxidação térmica na temperatura de $750^{\circ} \mathrm{C}$ por $10 \mathrm{~min}$ [117]. Esta camada de proteção causou uma pequena diminuição na condutividade térmica $(\lambda)$ do AlN de 198 para $185 \mathrm{~W} / \mathrm{mK}$. Sob condições hidrotérmicas, o AlN não teve degradação de sua resistência mecânica após exposição por 10 dias em água na temperatura de $180^{\circ} \mathrm{C}$ [118]. Porém, quando exposto ao vapor d'água, observou-se diminuição de $20 \%$ na resistência mecânica do AlN para as mesmas condições experimentais. $\mathrm{O}$ aumento da temperatura para $300{ }^{\circ} \mathrm{C}$ intensificou a degradação da resistência mecânica do AlN exposto à água e ao vapor d'água.

A presença de segundas fases de contorno de grão à base de silicatos, bem como o aumento de sua concentração na microestrutura de materiais cerâmicos aumenta a taxa de corrosão devido ao ataque preferencial dos contornos de grão [119-121]. No caso das cerâmicas de $\mathrm{Al}_{2} \mathrm{O}_{3}$, o processo de corrosão foi dependente da pureza do material, pois a presença de impurezas ou aditivos de sinterização intensificou a corrosão devido ao ataque preferencial das segundas fases de contorno de grão ricas em $\mathrm{SiO}_{2}$ e $\mathrm{CaO}$ [120]. Sabendo que os substratos de $\mathrm{Al}_{2} \mathrm{O}_{3}$ usados em microeletrônica possuem adições de compostos do sistema $\mathrm{SiO}_{2}-\mathrm{MgO}-\mathrm{CaO}$ [12], pode-se dizer que os substratos de AlN são mais resistentes à corrosão porque não utilizam aditivos de sinterização à base de silicatos, visto que reduzem significativamente o $\lambda$ [122]. Adicionalmente, observou-se que o AlN teve maior resistência à corrosão do que as cerâmicas de $\mathrm{Al}_{2} \mathrm{O}_{3}, \mathrm{Er}_{2} \mathrm{O}_{3}$ e $\mathrm{Y}_{2} \mathrm{O}_{3}$ em sistemas de sais fundidos, como o Flinak (46,5\%LiF-11,5\% NaF-42\% KF, \% em mol) [121]. Neste caso, cogitou-se a utilização do AlN como material adequado para o revestimento superficial dos materiais estruturais usados no sistema de cobertura de reatores de fusão refrigerados com sais fundidos. Embora haja poucos estudos sobre o comportamento de corrosão do AlN, os resultados analisados mostraram evidências de que o AlN tem maior resistência à corrosão do que $\mathrm{o}_{2} \mathrm{O}_{3} \mathrm{em}$ soluções usadas na fabricação de dispositivos eletrônicos.

\section{ÚlTIMAS TENDÊNCIAS EM PESQUISA DE NITRETO DE ALUMÍNIO}

Nos últimos anos, destacam-se três linhas distintas de pesquisas sobre a cerâmica de AlN. A primeira linha de pesquisa é caracterizada pela otimização de $\lambda$, similar ao observado nas décadas passadas, mas tendo como diferencial uma preocupação pelo comportamento mecânico. Mesmo com uma rota pré-estabelecida de processamento cerâmico, o AlN ainda tem estudos nas seguintes áreas: i) síntese de pós [123, 124]; ii) processamento cerâmico em meio aquoso $[125,126]$; e iii) sistemas multicomponentes de aditivos de sinterização $[38,57,94]$. De modo geral, o processo de sinterização tem sido conduzido em forno convencional sem aplicação de pressão (pressureless sintering), forno convencional com aplicação de pressão (hot pressing sintering) e forno com aplicação de pressão e aquecimento induzido por corrente elétrica (spark plasma sintering). Apesar dos significativos avanços em processamento nas últimas décadas, a primeira linha de pesquisa demostra que ainda existe preocupação pela otimização do processamento cerâmico do AlN. A dificuldade em processar a cerâmica de AlN foi apontada como um dos fatores restritivos à 
aceitação do AlN pela indústria de microeletrônica [19].

A segunda linha de pesquisa é caracterizada pela preparação de compósitos tanto cerâmicos $[6,57,112$, 127 129] como poliméricos [87, 130]. Em relação aos compósitos cerâmicos, observaram-se várias justificativas de estudos, tais como: i) compósitos AlN-BN, cujo objetivo é facilitar a usinagem do AlN por meio da adição de BN - usinagem de componentes com geometria complexa [112]; ii) compósitos AlN-TiN, cujo objetivo é montar módulos multicamadas em cerâmica para que o dispositivo eletrônico possa trabalhar acima de $500{ }^{\circ} \mathrm{C}$ [6]; iii) compósitos AlN-SiC, cujo objetivo é possibilitar a manipulação (tailoring) de propriedades elétricas e térmicas, para combinar as melhores propriedades de cada um dos referidos materiais [127]; iv) compósitos AlN-grafeno, cujo objetivo é aumentar a condutividade elétrica e resistência mecânica usando grafeno como material para preenchimento (filler) [128]; e v) compósitos AlNvidro, cujo objetivo é produzir substratos cerâmicos pela tecnologia de cerâmica cossinterizada a baixa temperatura (low temperature cofired ceramic, LTCC) [129]. Estes compósitos cerâmicos exibiram diminuição significativa de $\lambda$ e das propriedades mecânicas em comparação com a cerâmica monolítica de AlN. Já a utilização do AlN como material para preenchimento aumentou significativamente as propriedades térmicas e elétricas dos materiais de encapsulamento à base de polímero [87, 130].

A terceira linha de pesquisa é caracterizada pela síntese de AlN na forma de nanotubos [131], nanofios [132] e nanoagulhas (nano-needles) [133]. O processo de síntese do AlN em nanoestruturas não é recente [134], mas atualmente tem sido sintetizado em diversas morfologias (nanowhiskers, nanospheres, nanofibers, nanocones, nanorods, nanobelts, nanotips, nanorings) por meio de diferentes técnicas como sol-gel, sublimação, crescimento catalítico assistido por laser, deposição química a vapor e crescimento epitaxial por feixe molecular [132, 133]. Os resultados são promissores, pois o AlN nanoestruturado exibe um conjunto de propriedades que o torna um potencial candidato para a fabricação de dispositivos optoeletrônicos, lasers de alta energia, sensores de onda pulsante (pulse wave sensors), diodos emissores de luz (LED), ressonadores mecânicos em nanoescala e emissores de elétrons por campo [88, 133].

\section{MERCADO GLOBAL DO NITRETO DE ALUMÍNIO PARA APLICAÇÕES ELETRÔNICAS}

No início da década de 1990, a capacidade global de produção do pó de AlN foi estimada em 300 t/ano [17, $19,135]$, mas as vendas anuais atingiam apenas 140 t/ano [135]. As projeções indicavam um crescimento contínuo do mercado de AlN para aplicações eletrônicas, cujo valor total seria da ordem de 550 milhões de dólares para o ano 2000 $[13,17]$. Porém, o referido mercado arrecadou apenas 250 milhões de dólares em 2000, sendo estimado um consumo global de 200 t/ano de pó [136]. A franca expansão do mercado de AlN foi restringida, de modo geral, pelo elevado custo de produção do pó cerâmico, assim como pela baixa confiabilidade dos produtos manufaturados (inexperiência em processamento cerâmico) [19]. Apesar dos problemas citados, o mercado de pó de AlN cresceu de 140 para 200 t/ano durante a década de 1990. Recentemente, o Instituto de Pesquisa Yano divulgou uma pesquisa sobre o mercado global de materiais condutores térmicos [137]. Esta pesquisa estimou o tamanho de mercado em função dos valores de remessa dos produtos para os fornecedores, sendo o referido mercado dividido em três segmentos: materiais para preenchimento (filler), materiais para interface (sheets) e placas de circuito impresso. Apesar da baixa condutividade térmica $(\lambda), \mathrm{o} \mathrm{Al}_{2} \mathrm{O}_{3}$ liderou o mercado global de materiais para preenchimento em 2015, cujo volume de vendas correspondeu a $98,2 \%$ do mercado. O restante do mercado foi ocupado pelo nitreto de boro (BN) e AlN, que tiveram volumes de vendas relativos a $1,7 \%$ e $0,1 \%$ do mercado, respectivamente. As projeções para o ano de 2020 não mostraram mudanças significativas no mercado de materiais para preenchimento, sendo estimado para o AlN um aumento no consumo de 10 para 39 t/ano entre 2015 e 2020 , o que resultará em uma fatia de $0,4 \%$ do mercado.

Em relação ao mercado global de placas de circuito impresso, o alumínio liderou o mercado em 2015 ( $86 \%$ das vendas), seguido por $\mathrm{Al}_{2} \mathrm{O}_{3}(9 \%)$, $\mathrm{AlN}(4 \%)$ e $\mathrm{Si}_{3} \mathrm{~N}_{4}(1 \%)$. As projeções para o ano de 2020 mostram que o alumínio terá aumento no volume de vendas $(88,9 \%)$, enquanto os materiais cerâmicos apresentarão um recuo nas vendas, sendo previstos volumes de vendas de $8,1 \%$ para o $\mathrm{Al}_{2} \mathrm{O}_{3}$, $2,1 \%$ para o AlN e $0,9 \%$ para o $\mathrm{Si}_{3} \mathrm{~N}_{4}$. Assim, os volumes de vendas de substratos de AlN foram avaliados em 73000 e $53000 \mathrm{~m}^{2}$ para os anos de 2015 e 2020 , respectivamente. Comparando-se os volumes de vendas dos substratos de $\mathrm{AlN}$ e $\mathrm{Al}_{2} \mathrm{O}_{3}$, observou-se que o volume de vendas de $\mathrm{Al}_{2} \mathrm{O}_{3}$ foi 2,3 vezes maior $\left(170000 \mathrm{~m}^{2}\right)$ em 2015 e será 3,9 vezes maior $\left(207000 \mathrm{~m}^{2}\right)$ em 2020. Enfim, a pesquisa sobre o mercado global de materiais condutores térmicos mostrou que o AlN não substituiu o $\mathrm{Al}_{2} \mathrm{O}_{3}$ em aplicações eletrônicas, contrariando as previsões do início da década de 1990, que indicavam o AlN como principal material de gerenciamento térmico em dispositivos eletrônicos.

\section{CONSIDERAÇÕES FINAIS}

A cerâmica de AlN apresentou significativos avanços tecnológicos ao longo das últimas décadas, dos quais destacam-se: i) diminuição da temperatura de sinterização por meio do uso de aditivo de sinterização, que também resulta na otimização da condutividade térmica; ii) utilização de tratamento térmico pós-sinterização sob atmosfera redutora, que pode aumentar a condutividade térmica até ao redor de $270 \mathrm{~W} / \mathrm{mK}$, correspondendo a $84 \%$ da condutividade térmica do monocristal de AlN; iii) diminuição da temperatura de síntese do pó de AlN por meio da adição de agentes catalizadores, que pode resultar na redução de custos e na preparação de pós pré-aditivados (extinguindo o uso de aditivos de sinterização); iv) produção de pó resistente à água, que permite o processamento 
cerâmico em meio aquoso e favorece a estocagem do pó; e v) densificação em temperaturas tão baixas quanto $1600{ }^{\circ} \mathrm{C}$ por meio do uso de sistemas multicomponentes de aditivos de sinterização, que pode resultar em: a) condutividade térmica (150-170 W/mK) significativamente superior àquela observada para o $\mathrm{Al}_{2} \mathrm{O}_{3}(20-24 \mathrm{~W} / \mathrm{mK})$; e b) propriedades mecânicas $\left(\sigma_{\mathrm{f}}, \mathrm{K}_{\mathrm{Ic}}, \mathrm{E}\right.$ e $\left.\mathrm{H}_{\mathrm{V}}\right)$ relativamente próximas daquelas observadas para $\mathrm{O}_{2} \mathrm{O}_{3}$. Adicionalmente, o processo $\mathrm{DBC}$ foi estabelecido para o AlN, sendo observadas evidências de que o AlN pode ter maior resistência à corrosão do que $\mathrm{O}_{2} \mathrm{Al}_{2} \mathrm{O}_{3}$ em soluções ácidas e alcalinas empregadas no processamento de dispositivos eletrônicos. Estes avanços tecnológicos podem contribuir para desonerar os custos de produção, assim como adequar o AlN aos processos de fabricação de componentes passivos. Apesar dos resultados promissores, as rotas industriais não incorporaram a maioria dos referidos avanços tecnológicos e, com isso, os custos de produção não foram reduzidos suficientemente para tornar o AlN atrativo às indústrias de microeletrônica. As pesquisas de mercado demonstram que o AlN não substituiu $\mathrm{O}_{\mathrm{Al}_{2}} \mathrm{O}_{3}$ em aplicações eletrônicas envolvendo gerenciamento térmico. Porém, o mercado de AlN para aplicações eletrônicas pode ser expandido pela admissão de melhorias no processamento, que resultará na desoneração dos custos de produção e na fabricação de circuitos híbridos pela tecnologia HTCC. Outro fator que pode promover a ampliação do mercado de AlN é o desenvolvimento de nanoestruturas, que possibilitam novas aplicações, como dispositivos optoeletrônicos, lasers de alta energia, sensores de onda pulsante (pulse wave sensors), diodos emissores de luz (LED), ressonadores mecânicos em nanoescala e emissores de elétrons por campo. As cerâmicas de AlN, principalmente as densificadas em baixas temperaturas $\left(\leq 1600{ }^{\circ} \mathrm{C}\right)$, possuem propriedades mecânicas $\left(\mathrm{E}, \mathrm{H}_{\mathrm{v}}, \sigma_{\mathrm{f}} \mathrm{e}\right.$ $\mathrm{K}_{\mathrm{Ic}}$ ) relativamente próximas daquelas observadas para as cerâmicas de $\mathrm{Al}_{2} \mathrm{O}_{3}$. Portanto, a cerâmica de $\mathrm{AlN}$ pode ser empregada como material estrutural, mas sua disseminação tem sido restringida pelo elevado custo de produção em comparação com o $\mathrm{Al}_{2} \mathrm{O}_{3}$. Entretanto, a incorporação dos mencionados avanços em processamento cerâmico pode reduzir os custos de produção do AlN, tornando-o mais competitivo no mercado de materiais cerâmicos estruturais. Adicionalmente, o AlN tem sido considerado como um potencial material para aplicações em proteção balística, o que pode contribuir para sua inclusão no mercado de cerâmicas estruturais.

\section{REFERÊNCIAS}

[1] R. Roy, Key Eng. Mater. 123-124 (1996) 17.

[2] M. Entezarian, R.A.L. Drew, Mater. Sci. Eng. A212 (1996) 206.

[3] W. Werdecker, F. Aldinger, IEEE Trans. Compon. Packag. Technol. 7, 4 (1994) 399.

[4] D.D.L. Chung, Appl. Thermal Eng. 21 (2001) 1593.

[5] W.-H. Tuan, S.-K. Lee, J. Eur. Ceram. Soc. 34 (2014) 4117.

[6] Z. Valdez-Nava, S. Guillement-Fritsch, M. Ferrato, M.
Kozako, T. Lebey, Ceram. Int. 39 (2013) 8743.

[7] D. Jurków, J. Stiernsted, Ceram. Intern. 40 (2014) 10447.

[8] D. Jurków, J. Stiernsted, M. Dorczynski, G. Wetter, Ceram. Int. 41 (2015) 7860.

[9] A.B. Lostetter, F. Barlow, A. Elshabini, Microelectron. Reliab. 40 (2000) 365.

[10] H. He, R. Fu, D. Wang, X. Song, M. Jing, Mater. Lett. 61 (2007) 4131.

[11] J. Jarrige, T. Joyeux, J.P. Lecompte, J.C. Labbe, J. Eur. Ceram. Soc. 27, 2-3 (2007) 855.

[12] M. Pecht, R. Agarwal, F.P. McCluskey, T.J. Dishongh, S. Javadpour, R. Mahajan, Electronic packaging: materials and their properties, CRC Press, USA (1999) 30.

[13] Y. Baik, R.A.L. Drew, Key Eng. Mater. 122-124 (1996) 553.

[14] D. de Faoite, D. Browne, F.R. Chang-Díaz, K.T. Stanton, J. Mater. Sci. 47 (2012) 4211.

[15] G.A. Slack, R.A. Tanzilli, R.O. Pohl, J.W. Vandersande, J. Phys. Chem. Solids 48, 7 (1987) 641.

[16] R.-R. Lee, J. Am. Ceram. Soc. 74, 9 (1991) 2242.

[17] L.M. Sheppard, Am. Ceram. Soc. Bull. 69, 11 (1990) 1801.

[18] A. Franco, D.J. Shanafield, J. Mater. Sci.: Mater. Electron. 16, 3 (2005) 139.

[19] Am. Ceram. Soc. Bull. 72, 10 (1993) 26.

[20] B. Gil, Physics of wurtzite nitrides and oxides: passport to devices, Springer, Switzerland (2014) 2.

[21] S.A. Jang, G.M. Choi, J. Am. Ceram. Soc. 76, 4 (1993) 957.

[22] M.C. Wang, M.S. Tsai, N.C. Wu, J. Mater. Sci. 36, 13 (2001) 3283.

[23] N.C. Wu, M.S. Tsai, M.C. Wang, H.S. Liu, J. Cryst. Growth 208, 1-4 (2000) 189.

[24] E. Kroke, L. Loeffler, F.F. Lange, R. Riedel, J. Am. Ceram. Soc. 85, 12 (2002) 3117.

[25] N. Hashimoto, S. Deki, H. Yato, Y. Kanaji, J. Am. Ceram. Soc. 77, 6 (1994) 1633.

[26] H.U. Joo, W.-S. Jung, J. Mater. Process. Technol. 204 (2008) 498.

[27] G. Selvaduray, L. Sheet, Mater. Sci. Technol. 9, 6 (1993) 463.

[28] P. Lefort, M. Billy, J. Am. Ceram. Soc. 76, 9 (1993) 2295.

[29] Y. Kameshima, M. Irie, A. Yasumori, K. Okada, J. Eur. Ceram. Soc. 24, 15-16 (2004) 3801.

[30] J.W. Li, M. Nakamura, T. Shirai, K. Matsumaru, C. Ishizaki, K. Ishizaki, J. Am. Ceram. Soc. 89, 3 (2006) 937.

[31] T. Ide, K. Komeya, T. Meguro, J. Tatami, J. Am. Ceram. Soc. 82, 11 (1999) 2993.

[32] A.L. Molisani, H.N. Yoshimura, Mater. Res. Bull. 45 (2010) 733.

[33] P. Bowen, J.G. Highfield, A. Mocellin, T.A. Ring, J. Am. Ceram. Soc. 73, 3 (1990) 724.

[34] I. Ganesh, N. Thiyagarajan, G. Sundararajan, S.M. Olhero, J.M.F. Ferreira, J. Eur. Ceram. Soc. 28 (2008) 2281. [35] X.J. Luo, B.L. Zhang, W.L. Li, H.R. Zhuang, Ceram. Int. 30 (2004) 2099. 
[36] S.M. Olhero, Z.S. Jensen, M.A. Einarsud, T. Grande, J.M.F. Ferreira, J. Am. Ceram. Soc. 90, 11 (2007) 3589.

[37] X.J. Luo, J. Li, B. Zhang, W. Li, H. Zhuang, J. Am. Ceram. Soc. 89, 3 (2006) 836.

[38] M. Lee, D.K. Kim, J. Eur. Ceram. Soc. 34, 15 (2014) 3627.

[39] R.A.L. Drew, Y. Baik, M. Entezarian, Mater. Sci. Forum 325-326 (2000) 249.

[40] Q. Li, Z. Wang, C. Wu, X. Cheng, J. Alloys Compd. 640 (2015) 275.

[41] J.Y. Qiu, Y. Hotta, K. Watari, K. Mitsuishi, M. Yamazaki, J. Eur. Ceram. Soc. 26 (2006) 385.

[42] A.L. Molisani, H.N. Yoshimura, H. Goldenstein, J. Eur. Ceram. Soc. 26, 15 (2006) 3431.

[43] K. Komeya, H. Inoue, A. Tsuge, Yogyo-Kyokai-Shi 89, 6 (1981) 330 .

[44] K. Komeya, A. Tsuge, H. Inoue, H. Ohta, J. Mater. Sci. Lett. 1 (1982) 325.

[45] S. Prochazka, C.F. Bobik, in Proc. $5^{\text {th }}$ Int. Conf. Sintering Related Phenomena, Ed. G.C. Kuczynski, Mater. Sci. Res., Indiana (1979) 321.

[46] K.A. Schwetz, H. Knoch, A. Lipp, Progess in nitrogen ceramics, Ed. F.L. Riley, Falmer, U.K. (1981) 245.

[47] A.V. Virkar, T.B. Jackson, R.A. Cutler, J. Am. Ceram. Soc. 72, 11 (1989) 2031.

[48] N. Kuramoto, H. Taniguchi, I. Aso, Am. Ceram. Bull. $\mathbf{6 8 ,} 4$ (1989) 883.

[49] A.C. da Cruz, R.J. Munz, H. Vali, J. Mater. Sci. Lett. 17 (1998) 1255.

[50] M.C. Wang, C.K. Hong, M.S. Tsai, N.C. Wu, J. Ceram. Soc. Jpn. 108, 10 (2000) 869.

[51] N. Hashimoto, H. Yoden, J. Am. Ceram. Soc. 75, 8 (1992) 2098.

[52] K. Watari, M.E. Brito, M. Yasuoka, M.C. Valecillos, S. Kanzaki, J. Ceram. Soc. Jpn. 103, 9 (1995) 891.

[53] K. Watari, H.J. Hwang, M. Toriyama, S. Kanzaki, J. Mater. Res. 14, 4 (1999) 1409.

[54] K. Watari, M.C. Valecillos, M.E. Brito, M. Toriyama, S. Kanzaki, J. Am. Ceram. Soc. 79, 12 (1996) 3103.

[55] Y.C. Liu, H. Zhou, L. Qiao, Y. Wu, J. Mater. Sci. Lett. 18 (1999) 703.

[56] L.A. Qiao, H.P. Zhou, H. Xue, S.H. Wang, J. Eur. Ceram. Soc. 23, 1 (2003) 61.

[57] R. Kobayashi, K. Oh-Ishi, R. Tu, T. Goto, Ceram. Int. 41 (2015) 1897.

[58] T. Sakai, M. Kuriyama, T. Inukai, T. Kizima, YogyoKyokai-Shi 86, 4 (1978) 174.

[59] T. Sakai, M. Iwata, J. Mater. Sci. 12, 8 (1997) 1659.

[60] A.L. Molisani, H.N. Yoshimura, H. Goldenstein, J. Mater. Sci. Mater. Electron. 20 (2009) 1.

[61] A.L. Molisani, H.N. Yoshimura, H. Goldenstein, Cerâmica 52, 322 (2006) 151.

[62] A.L. Molisani, H. Goldenstein, H.N. Yoshimura, Cerâmica 56, 340 (2010) 331.

[63] M. Kasori, F. Ueno, J. Eur. Ceram. Soc. 15, 5 (1995) 435.

[64] A. Geith, M. Kulig, T. Hofmann, C. Russel, J. Mater.
Sci. 28 (1993) 865.

[65] J. Jarrige, K. Bouzouita, C. Doradoux, M. Billy, J. Eur. Ceram. Soc. 12, 4 (1993) 279.

[66] T.B. Troczynski, P.S. Nicholson, J. Am. Ceram. Soc. 72, 8 (1989) 1488.

[67] T. Nakamatsu, F. Pomar, K. Ishizaki, J. Mater. Sci. 34 (1999) 1553.

[68] K. Watari, M. Kawamoto, K. Ishizaki, J. Mater. Sci. 26 (1991) 4727.

[69] H. Nakano, K. Watari, K. Urabe, J. Am. Ceram. Soc. 23, 10 (2003) 1761.

[70] H. Nakano, K. Watari, H. Hayashi, K. Urabe, J. Am. Ceram. Soc. 85, 12 (2002) 3093.

[71] K. Watari, H. Nakano, K. Urabe, K. Ishizaki, S.X. Cao, K. Mori, J. Mater. Res. 17, 11 (2002) 2940.

[72] H. Buhr, G. Muller, J. Eur. Ceram. Soc. 12, 4 (1993) 271.

[73] K. Watari, J. Ceram. Soc. Jpn. 109, 1 (2001) S7.

[74] Y. Kurokawa, K. Utsumi, H. Takamizawa, J. Am. Ceram. Soc. 71, 7 (1988) 588.

[75] H. Yan, W.R. Cannon, D.J. Shanefield, J. Am. Ceram. Soc. 76, 1 (1993) 166.

[76] S. Mitra, G. Dutta, I. Dutta, J. Am. Ceram. Soc. 78, 9 (1995) 2335.

[77] E. Hagen, Y.D. Yu, T. Grande, R. Hoier, M.A. Einarsrud, J. Am. Ceram. Soc. 85, 12 (2002) 2971.

[78] K. Komeya, J. Tatami, Mater. Sci. Forum 554 (2007) 181.

[79] A. Bellosi, L. Esposito, E. Scafe, L. Fabbri, J. Mater. Sci. 29 (1994) 5014.

[80] T.B. Jackson, A.V. Virkar, K.L. More, R.B. Dinwiddie Jr, R.A. Cutler, J. Am. Ceram. Soc. 80, 6 (1997) 1421.

[81] J. Jarrige, J.P. Lecompte, J. Mullot, G. Muller, J. Eur. Ceram. Soc. 17, 15-16 (1997) 1891.

[82] A. Berger, J. Am. Ceram. Soc. 78, 1 (1995) 153.

[83] J.H. Harris, R.A. Youngman, J. Mater. Res. 5, 8 (1990) 1763.

[84] K. Watari, H.J. Hwang, M. Toriyama, S. Kanzaki, J. Am. Ceram. Soc. 79, 7 (1996) 1979.

[85] P.S. de Baranda, A.K. Knudsen, E. Ruh, J. Am. Ceram. Soc. 77, 7 (1994) 1846.

[86] S. Pietranico, S. Pommier, S. Lefebvre, S. Pattofatto, Int. J. Fatigue 31 (2009) 1911.

[87] R.K. Goyal, A.N. Tiwari, Y.S. Negi, Composites Part B 47 (2013) 70.

[88] A.T. Wieg, Y. Kodera, Z. Wang, C. Dames, J.E. Garay, Acta Materialia 86 (2015) 148.

[89] M. Azzaz, A. George, Mater. Sci. Eng. A 364 (2004) 84.

[90] H.N. Yoshimura, A.L. Molisani, N.E. Narita, P.F. Cesar, H. Goldenstein, Mater. Res. 10, 2 (2007) 127.

[91] P.S. Branicio, R.K. Kalia, A. Nakano, P. Vashishta, F. Shimojo, J. Rino, J. Mech. Phys. Solids 56 (2008) 1955.

[92] G. Subhash, G. Ravichandran, J. Mater. Sci. 33 (1998) 1933.

[93] Z. Shi, S. Chen, J. Wang, G. Qiao, Z. Jin, J. Eur. Ceram. Soc. 31 (2011) 2137. 
[94] T. Kusunose, T. Sekino, Ceram. Int. 42, 11 (2016) 13183.

[95] F.M. Xu, Z.J. Zhang, X.L. Shi, Y. Tan, J.M. Yang, J. Alloys Compd. 509 (2011) 8688.

[96] Q. Li, Z. Wang, C. Wu, X. Cheng, J. Alloys Compd. 640 (2015) 275.

[97] F. Roulet, P. Tristant, J. Desmaison, R. Rezakhanlou, M. Ferrato, J. Eur. Ceram. Soc. 17, 15-16 (1997) 1877.

[98] A.H. Lubis, N.L. Hecht, G.A. Graves Jr, R. Ruh, J. Am. Ceram. Soc. 82, 9 (1999) 2481.

[99] R. Terao, J. Tatami, T. Meguro, K. Komeya, J. Eur. Ceram. Soc. 22, 7 (2002) 1051.

[100] S. Dagdag, J. Alexis, J.D. Beguin, J.A. Petit, M. Ferrato, Sintering 05, France (2005) 168.

[101] L.M. Braun, S.J. Bennison, B.R. Lawn, J. Am. Ceram. Soc. 75, 11 (1992) 3049.

[102] T. Sakai, J. Am. Ceram. Soc. 61, 9 (1978) 460.

[103] H.N. Yoshimura, A.L. Molisani, G.R. Siqueira, A.C. de Camargo, N.E. Narita, P.F. Cesar, H. Goldenstein, Cerâmica 51, 319 (2005) 239.

[104] S.R. Witek, G.A. Miller, M.P. Harmer, J. Am. Ceram. Soc. 72, 3 (1989) 469.

[105] S. Wei, Z. Xie, W. Xue, Z. Yi, J. Chen, L. Cheng, Mater. Lett. 119 (2014) 32.

[106] S. Wei, Z. Xie, W. Xue, Z. Yi, J. Chen, Ceram. Int. 40, 8 (2014) 13715.

[107] R.W. Rice, J. Mater. Sci. 31 (1996) 1509.

[108] Y.B.P. Kwan, D.J. Stephenson, J.R. Alcock, J. Mater. Sci. 35 (2000) 1205.

[109] H. Abe, K. Sato, M. Naito, K. Nogi, T. Hotta, J. Tatami, K. Komeya, Powder Technol. 159, 3 (2005) 155.

[110] I.L. Tangen, Y.D. Yu, T. Grande, R. Hoier, M.A. Einarsrud, Ceram. Int. 30 (2004) 931.

[111] J.L. Huang, J.M. Jih, J. Am. Ceram. Soc. 79, 5 (1996) 1262.

[112] C. Jin, T. Wang, L. Pan, J. Yang, C. Hu, T. Qiu, J. Mater. Sci. Mater. Electron. 27, 2 (2016) 2014.

[113] T. Ostrowski, A. Ziegler, R.K. Bordia, J. Rodel, J. Am. Ceram. Soc. 81 (1998) 1852.

[114] P. Boch, J.C. Glandus, J. Jarrige, J.P. Lecompte, J. Mexmain, Ceram. Int. 8, 1 (1982) 34.

[115] D. Gerlich, S.L. Dole, G.A. Slack, J. Phys. Chem. Solids 47, 5 (1986) 437.

[116] G. Hu, C.Q. Chen, K.T. Ramesh, J.W. McCauley, Acta Mater. 60 (2012) 3480.
[117] L.M. Svedberg, K.C. Arndt, M.J. Cima, J. Am. Ceram. Soc. 83, 1 (2000) 41.

[118] H. Tamai, T. Hamauzu, A. Ryumon, K. Aoki, K. Oda, T. Yoshio, J. Am. Ceram. Soc. 83, 12 (2000) 3216.

[119] M. Schacht, N. Boukis, E. Dinjus, J. Mater. Sci. 35 (2000) 6251.

[120] T. Watanabe, M. Kondo, T. Nagasaka, A. Sagara, J. Plasma Fusion Res. 9 (2010) 342.

[121] K.R. Mikeska, S.J. Bennison, S.L. Grise, J. Am. Ceram. Soc. 83, 5 (2000) 1160.

[122] P.S. de Baranda, A.K. Knudsen, E. Ruh, J. Am. Ceram. Soc. 76, 7 (1993) 1761.

[123] H.H. Nersisyan, B.U. Yoo, T.H. Lee, J.H. Lee, J. Am. Ceram. Soc. 98, 12 (2015) 3740.

[124] W.-S. Jung, Ceram. Int. 42, 13 (2016) 14716.

[125] A. Kocjan, A. Dakskobler, K. Krnel, T. Komac, J. Eur. Ceram. Soc. 31 (2011) 815.

[126] L. Guo, J. Yang, Y. Feng, T. Qiu, B. Chen, W. Wan, Ceram. Int. 42, 7 (2016) 8066.

[127] K.J. Kim, Y.-W. Kim, K.-Y. Lim, T. Nshimura, E. Narimatsu, J. Eur. Ceram. Soc. 35 (2015) 2715.

[128] C. Yun, Y. Feng, T. Qiu, J. Yang, X. Li, L. Yu, Ceram. Int. 41, 7 (2015) 8643.

[129] S. Liu, H. Zhang, B. Zhang, X. Zhong, J. Ma, F. Ye, Ceram. Int. 42 (2016) 7253.

[130] Y. Zhou, H. Wang, L. Wang, K. Yu, Z. Lin, L. He, Y. Bai, Mater. Sci. Eng. B 177 (2012) 892.

[131] J. Beheshtian, M. Baei, Z. Bagheri, A.A. Peyghan, Microelectron. J. 43 (2012) 452.

[132] Kenry, K.-T. Yong, S.F. Yu, J. Mater. Sci. 47 (2012) 5341 .

[133] S.H. Shah, G. Nabi, W. Khan, A. Majid, C. Cao, S. Ali, M. Hussain, A. Nabi, S. Ishaq, F. Butt, Mater. Lett. 107 (2013) 255.

[134] Q. Wu, Z. Hu, X. Wang, Y. Lu, K. Huo, S. Deng, N. Xu, B. Shen, R. Zhang, Y. Chen, J. Mater. Chem. 13 (2003) 2024.

[135] G. MacZura, K.J. Moody, E.M. Anderson, M.K. Kunka, Am. Ceram. Soc. Bull. 74, 6 (1995) 97.

[136] T.J. Mroz, Am. Ceram. Soc. Bull. 79, 6 (2000) 45.

[137] Global thermal conductive materials market: key research findings 2016, disponível: <http://www. yanoresearch.com/press/press.php/001540>, acesso: 15/10/2016.

(Rec. 17/08/2016, Rev. 04/11/2016, 02/01/2017, Ac. 16/02/2017) 